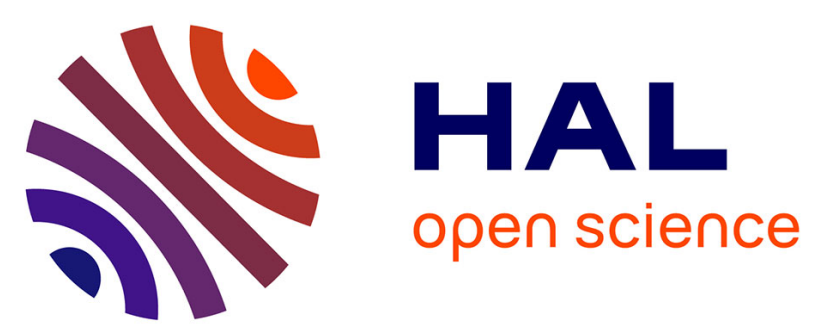

\title{
Membrane Curvatures and Stress-strain Full Fields of Axisymmetric Bulge Tests from 3D-DIC Measurements. Theory and Validation on Virtual and Experimental results
}

Guilherme Machado, Denis Favier, Grégory Chagnon

\section{To cite this version:}

Guilherme Machado, Denis Favier, Grégory Chagnon. Membrane Curvatures and Stress-strain Full Fields of Axisymmetric Bulge Tests from 3D-DIC Measurements. Theory and Validation on Virtual and Experimental results. Experimental Mechanics, 2012, 52 (7), pp.865-880. 10.1007/s11340-0119571-3 . hal-01974147

\section{HAL Id: hal-01974147 \\ https://hal.science/hal-01974147}

Submitted on 10 Jan 2019

HAL is a multi-disciplinary open access archive for the deposit and dissemination of scientific research documents, whether they are published or not. The documents may come from teaching and research institutions in France or abroad, or from public or private research centers.
L'archive ouverte pluridisciplinaire HAL, est destinée au dépôt et à la diffusion de documents scientifiques de niveau recherche, publiés ou non, émanant des établissements d'enseignement et de recherche français ou étrangers, des laboratoires publics ou privés. 


\title{
Membrane curvatures and stress-strain full fields of axisymmetric bulge tests from 3D-DIC measurements. Theory and validation on virtual and experimental results.
}

\author{
Guilherme Machado • Denis Favier · Grégory Chagnon
}

\begin{abstract}
The bulge test is mostly used to analyze equibiaxial tensile stress state at the pole of inflated isotropic membranes. Three-dimensional digital image correlation (3D-DIC) technique allows the determination of three-dimensional surface displacements and strain fields. In this paper, a method is proposed to determine also the membrane stress tensor fields for in-plane isotropic materials, independently of any constitutive equation. Stress-strain state is then known at any surface point which enriches greatly experimental data deduced from the axisymmetric bulge tests. Our method consists, first in calculating from the 3D-DIC experimental data the membrane curvature tensor at each surface point of the bulge specimen. Then, curvature tensor fields are used to investigate axisymmetry of the test. Finally in the axisymmetric case, membrane stress tensor fields are determined from meridional and circumferential curvatures combined with the measurement of the inflating pressure. Our method is first validated for virtual 3D-DIC data, obtained by numerical simulation of a bulge test using a hyperelastic material model. Afterward, the method is applied to an experimental bulge test performed using as material a silicone elastomer. The stress-strain fields which are obtained using the proposed method are compared with results of the finite element
\end{abstract}

G. Machado · D. Favier · G. Chagnon

Université de Grenoble/CNRS, Laboratoire 3SR

BP53, 38041 Grenoble Cedex 9, France

E-mail: denis.favier@hmg.inpg.fr

G. Machado

E-mail: guilherme.machado@hmg.inpg.fr

G. Chagnon

E-mail: gregory.chagnon@hmg.inpg.fr 
multiaxial stress and strain fields [6], to study the mechanical properties of human tissues $[7,8]$ and to measure the finite load-deformation behavior of a living cells sheet cultured on a mesoscopic freestanding elastomer membrane [9]. More recently Grolleau et al. [10] combined a Hopkinson bar system with a conventional bulge test to study the biaxial testing of sheet materials at high strain rates.

On the theoretical point of view, the bulge test was as well studied in detail. A seminal work was proposed by Adkins and Rivlin [11] that details the boundary value problem, in the field of nonlinear elasticity, associated with the Mooney isotropic membrane inflation. Other important work has come from Hill [12] introducing a theoretical analysis of plastic flow at the pole of a metal diaphragm, based on the Lévy-Mises stress-strain equations. In an analogous manner, Ross and Prager [13] showed that the use of Tresca's yield condition and associated flow rule leads to a simple theory, where the basic equations can be integrated in closed form even for finite deflections.

Bulge test was mostly conducted as an easy way to determine mechanical behavior of isotropic materials subjected to equibiaxial tensile stress state. Indeed, due to the axial symmetry of the problem and respecting the primary assertions of the membrane theory, the equibiaxiality of the stress and strain is obtained at the top of the inflated sample. Found over the pole neighborhood, where a spherical cap is supposed to exist, the equibiaxial stress-strain state can be easily calculated. To achieve this goal the knowledge of the curvature radius, tangential elongation and pressure recorded during the test are needed. Such approach was recently used to study rubber-like materials $[14,15]$, and NiTi superelastic sheets [16]. However, the current shape of the total bulge is rarely spherical, as shown by Dudderar et al. [17] using the two-source holographic technique. Therefore, if the pressure and principal curvatures fields are measured, much more information is available from axisymmetrical bulge test than only stress and strain states at the pole. The membrane stress tensor can be determined from these data using membrane theory and equilibrium equations without defining any explicit constitutive equation. However, the accurate experimental measurement of curvatures is not a simple task. To avoid this difficulty, a relation describing the material behavior was often used in the past. In this case, the principal curvatures were often written in terms of the principal stretch ratios and replaced in the Lagrangian form of the equilibrium equations. In a general manner, this procedure intends to reduce the problem to a first-order system by introducing new variables $[18,19]$. As a consequence, there is also loss in generality in assuming an explicit 
form for the constitutive relation which is chosen to represent the material. See for example the works [20] for hyperelastic potentials; [21] and [22] for viscoelastic behavior.

In a classical manner, the bulge specimen is supposed to be deformed axisymmetrically and profiles are measured. In that case, meridional curvature is directly measured and circumferential curvature is deduced under axisymmetric assumption [23]. The accuracy of this process heavily depends on the finite number of experimental measurements points, and information data is restricted to a meridional path only. This manner to compute the principal curvatures is restricted to axisymmetric problems and also a numerical procedure must be employed to solve the coupled nonlinear differential problem.

In this context, the present work intends to provide useful method to obtain the membrane stress and strain fields during the bulge test using the three-dimensional digital image correlation (3D-DIC) technique. The 3D-DIC is able to capture three-dimensional surface geometry and displacements of the deformed surface. This paper explains how these kinematical fields allow to evaluate the strain and curvatures fields in each point of the membrane. The stress field can also be deduced using the axisymmetrical hypothesis. Section 2 starts with a brief description of 3D-DIC technique, where the bases of contour and strain measurement principles are outlined. In the same section, the theoretical framework to evaluate the first and second fundamental forms of surface and relations between principal curvatures and membrane stress are emphasized: a method to determine the curvature fields from 3D-DIC data is thus presented. Then we show that curvature fields combined with the measurements of the inflation pressure allow calculating the stress fields for an axisymmetric bulge. In Section 3, a finite element calculation is realized on a Mooney hyperelastic membrane to emulate experimental data, i.e., generate a virtual 3D-DIC information. These data allow to validate the curvature calculation method and to estimate errors associated with stress determination. In Section 4 the method is applied to real experimental data. The experimental results obtained in the bulge test of a silicone rubber-like material are presented. All precautions concerning the experimental setup are emphasized. The tridimensional correlation process is not perfect. In view of missing and outlying values that can occasionally occur, a strategy is invoked to lead to these occurrences. Finally, the experimental data fields are compared to a finite element model carried out using a neo-Hookean hyperelastic law fitted on uniaxial and equibiaxial tensile tests. This allows to highlight the advantages of the proposed method compared to usual methods used to analyze bulge tests. 


\section{Theoretical framework}

\subsection{A brief outline of 3D-DIC}

In the 3D-DIC technique, random gray-value dot patterns on specimen surfaces are observed by two cameras in a stereoscopic setup. Also called stereo digital image correlation (SDIC), the 3DDIC is based on both stereo-vision technique and digital image correlation (DIC). A comprehensive description of metrological aspects of 3D-DIC technique can be found in the literature, e.g. [24], [26] and [27]. See [28] for references on applications of stereo-vision and 3D-DIC to experimental mechanics.

The stereo-vision technique allows to recover the three-dimensional information of a scene from two different view points. Considering a physical point $M$ of coordinates $X^{i}$ in a Cartesian reference frame to be measured (Fig. 1). Forward, Latin indices represent the values 1, 2, 3 and, the Greek ones the values 1,2 . The stereo-projections of the point $M\left(X^{i}\right)$ on the images planes of two cameras $C 1$ and $C 2$ are given by $M_{C 1}\left(X_{S 1}^{\alpha}\right), M_{C 2}\left(X_{S 2}^{\alpha}\right)$, where $X_{S}^{\alpha}$ are the camera sensor coordinate system. Every deviation from the straightness of the projection can be related to the presence of radial and tangential distortions. Fig. 1 illustrates the epipolar geometry, i.e., the geometry of stereo vision defined by the pinhole positions $C_{\alpha}$, focal lengths $f_{\alpha}$, and image plane centers $P_{\alpha}$. It is convenient to define a set of coordinate systems to represent the imaging process. The first coordinate system is the global coordinate system $(G C S)$, with axes $\left(X^{1}, X^{2}, X^{3}\right)$. The second coordinate system typically defined is the camera coordinate system $(C C S)$ located at the pinhole positions $C_{\alpha}$. The third set of axes defines the sensor coordinate system $(S C S)$, with $X_{S}^{1}, X_{S}^{2}$ in terms of pixels.

The 3D-DIC uses a DIC algorithm to determine point correspondences between two images of a specimen acquired at the same time from two bounded cameras. The digitized images are compared to match subsets - so called facets - from one image to another. The facet is a square area $f_{\text {size }} \times f_{\text {size }}$ pixels, centered on the evaluation grid point. This facet is used from the correlation algorithm in order to identify the corresponding area in the second image. The correlation scores are computed by measuring the similarity of a fixed facet in the first image to a shifting subset window in the second one. Knowing of the imaging parameters for each camera and the orientations of the cameras with respect to each other by a calibration procedure, the position of each object point in three dimensions is calculated. The minimal facet size $\left(f_{\text {size }}\right)$ is limited by the size and 
roughness of the stochastic pattern on the object surface. Each facet must contain a characteristic part of the pattern with good contrast features.

As a result, the 3D-DIC provides a set of physical points $M$ in terms of Cartesian coordinates $X^{i}$ in a global reference system, arbitrarily defined during the calibration phase. The calibration, in the commercial system used in this work, is done by taking a series of exposures of a calibration target with both cameras simultaneously, i.e., both cameras are calibrated as a stereo rig. The calibration target is a chess pattern with known geometry. This target is put successively into different spatial orientations with respect to the cameras sensors. The software detects the corners of the squares. Additional circular markers placed on the target surface define the center and the orientation of the calibration target. The evaluation algorithm then calculates the intrinsic parameters (focal length $f$, image plane center $P$, radial and tangential distortion parameters) for each camera. Intrinsic parameters are influenced by focal lens, focal distance and aperture. These parameters are considered as intrinsic since the cameras remain in fixed positions relative to each other during the experiment. The evaluation algorithm computes the extrinsic parameters (translation vector and the rotation matrix between the GCS and the SCS) as well, which in turn are influenced by the intrinsic parameters and the positions of the cameras. The uncertainties of the calibration parameters, resulting from the deviations of the detected markers from the model positions are then calculated. A total number of eight images are typically sufficient to calculate all calibration parameters accurately [25]. A comprehensive description of the 3D-DIC calibration method with the mathematical development can be found in [26].

Note that the spatial resolution $\left(S_{\text {res }}\right)$ of the discretized surface depends essentially on camera sensor resolution $\left(C_{r e s}\right)$ and on the choice of the evaluation grid that defines the distance between the data points on the object. The grid spacing $\left(G_{s}\right)$ is the distance between the grid points in pixel. Thus, grid spacing limits the spatial resolution, as each grid point represents one single data point of the result. Finally, data output is arranged in a matrix representation in the form $X^{i}(u, v)$ where the integer values $u, v \in\left[1,\left\lfloor C_{r e s} / G_{s}\right\rceil\right]$ are respectively lines and columns indices of the matrix representation for each coordinate $\left(X^{i}\right)$. See the scheme in Fig. 1.

Once the 3D contour has been determined, the next step in digital 3D correlation is the determination of the three-dimensional deformation of the object surface. This process is carried out by correlation of the images, taken by both cameras in the deformed state, with their original reference images. To evaluate the 3D displacement field, 3D-DIC determines point correspondences 


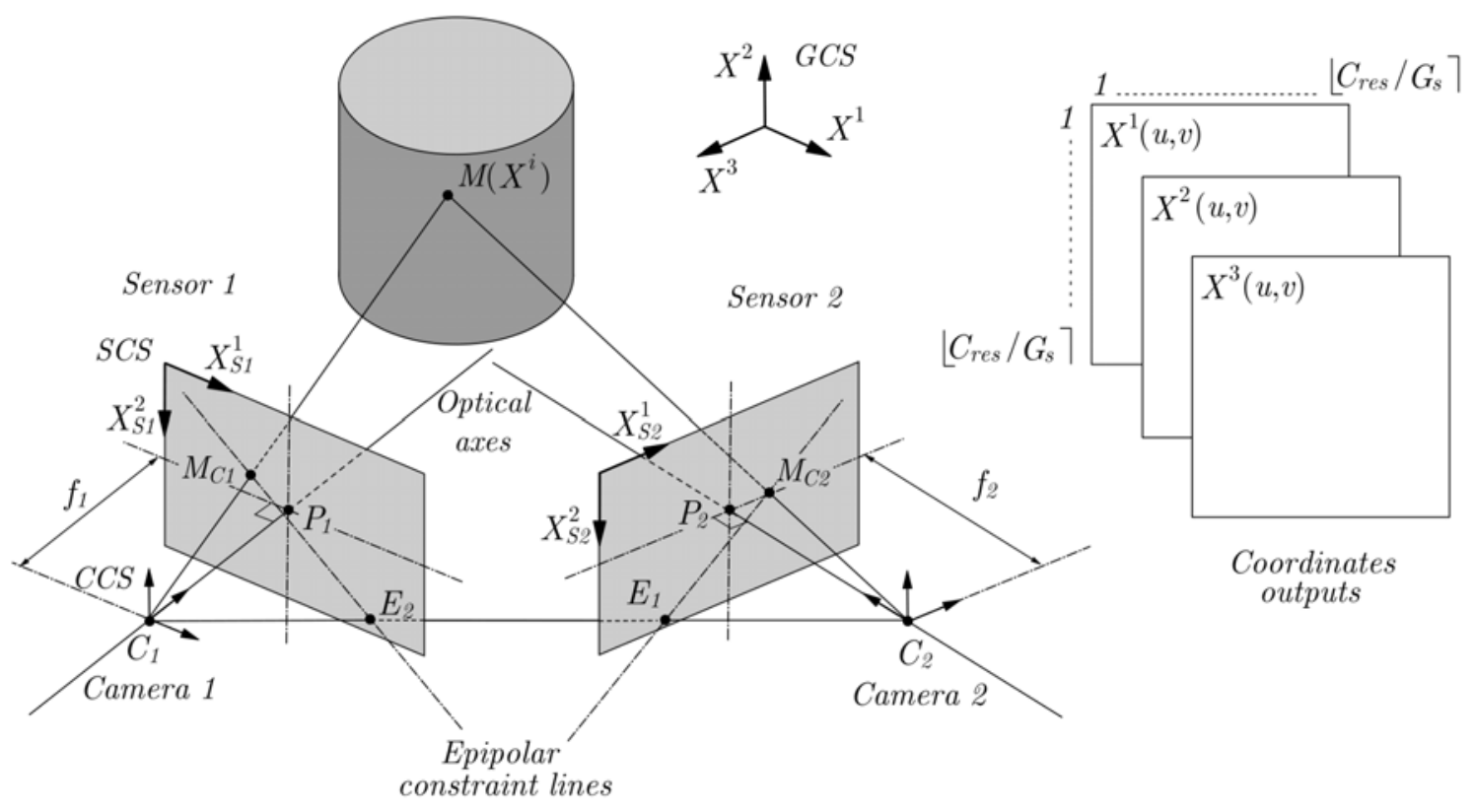

Fig. 1 Representation of the epipolar geometry and the coordinates output format.

between the stereo pairs acquired before and after deformation. The correlation algorithm tracks the observed gray value patterns for each camera and transforms corresponding facet positions in both cameras into 3D coordinates for each deformation step, resulting in a track of each surface facet in $3 \mathrm{D}$ space and over time. With the known position vectors of each surface point and the reference contour, the strains can be determined by calculating the gradient of the deformation or by the distortion of the facets, i.e., strain can be computed either directly by differentiation of the displacements of adjacent surface points or measured pointwise by analyzing the distortion of each local facet which was used for correlation [26, 27].

\subsection{Errors sources on the 3D-DIC}

The evaluation of a 3D correlation measurement results in $3 \mathrm{D}$ coordinates, displacements and inplane strains on the specimen surface. The corresponding data uncertainties originate from different sources, which can be divided into three categories [25]: statistical correlation errors, systematical correlation errors and 3D reconstruction errors. Statistical correlation errors are principally related to the limited number of pixels and corresponding gray values in each facet, and finally by the 
fact that the facet position has to be determined with subpixel accuracy. Additional potential error sources are statistical noise of the gray values, different illumination conditions for the two cameras, image contrast and size of the speckle pattern on the specimen surface. Systematical correlation errors are introduced by subpixel effects. They might occur if the facet transformation model used is not appropriate to describe the complexity of the real transformation, e.g., in a curved specimen surface or for significant lens distortions [29]. Systematical errors is one of the principal limitation for the resolution of the resulting data [25]. Finally, uncertainties of the calibration parameters lead to errors when reconstructing the 3D coordinates. Calibration errors appear in a systematical manner as a function of the facet positions in the camera frames, causing local distortions of the reconstructed 3D space. However, results from multiple experiments performed in [26] indicate a typical accuracy for the displacement field on curved or planar specimens on the order of \pm 0.01 pixels for the in-plane components and $D / 50000$ in the out-of-plane component (where $D$ is the distance from the object to the camera) for typical stereo-camera arrangements. A point-to-point accuracy of $\pm 10^{-4}$ for the in-plane surface strains is achievable even when the object is subjected to large rigid-body rotations and arbitrary amounts of rigid-body translations since these motions do not corrupt the strain measurements.

2.3 Curvature evaluation from coordinates fields measured by 3D-DIC

Using the 3D-DIC, the reference surface $\Omega_{0}$ of the object in question is discretized by a regular grid that represents the surface curvilinear coordinates $\Theta^{\alpha}$. These coordinates are obtained directly from the grid placed by 3D-DIC using the grid indexes $\Theta^{1}=u$ and $\Theta^{2}=v$ where $u$ and $v$ are respectively lines and columns indices of the matrix representation $X^{i}(u, v)$. This grid maps any discrete point $M_{0}$ of the undeformed body, associating a set of curvilinear coordinates, so called $\Theta^{\alpha}-$ curves. Since the coordinates $\Theta^{\alpha}$ are convective, these curves are subjected to the same deformation as the body and are transformed at time $t$ in a new set of curves that represents the deformed state $\Omega_{t}$, as presented in Fig. 2. In the same figure, an arbitrary surface in three-dimensional Euclidean space $\mathbb{E}^{3}$ is considered. The mapping of $\Omega_{0}$ into $\Omega_{t}$ is such that the correspondence of the points $M_{0}$ and $M$ is assumed one to one. The reference and deformed surfaces are defined by $\mathbf{S}=\mathbf{S}\left(\Theta^{\alpha}\right)$ and $\mathbf{s}=\mathbf{s}\left(\Theta^{\alpha}, t\right)$, respectively, with $\mathbf{S}$ and $\mathbf{s} \in \mathbb{E}^{3}$, where $\Theta^{\alpha}$ are the surface curvilinear coordinates. In 
the following, s is supposed to be single-valued and sufficiently differentiable with respect to both arguments $\Theta^{\alpha}$ as many times as required.

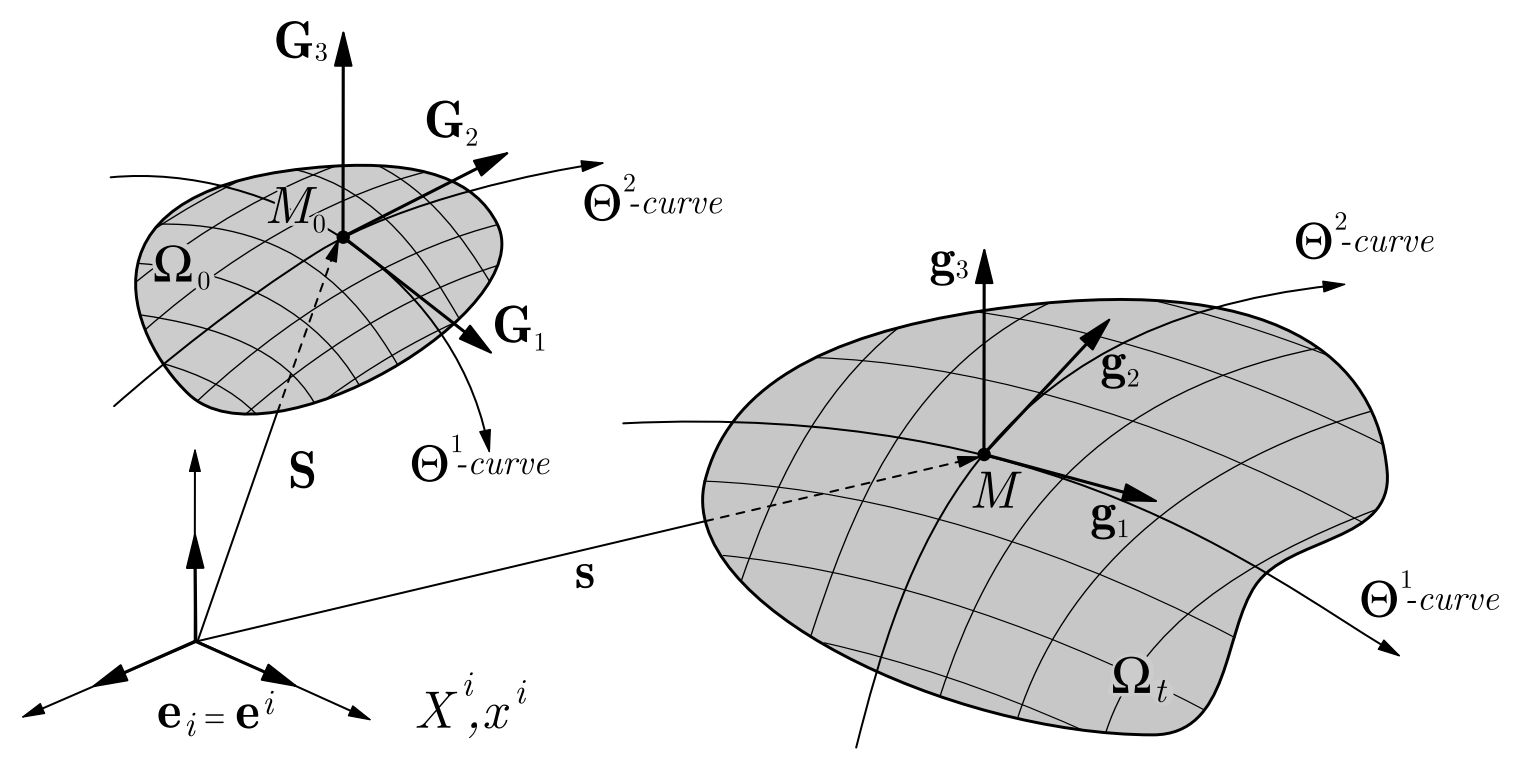

Fig. 2 The surface basic geometric variable definition: $\mathbf{e}^{i}$ are the unit base vectors related with the orthogonal Cartesian coordinates $X^{i}$ and $x^{i} ; \mathbf{G}_{i}$ and $\mathbf{g}_{i}$ are the covariant basis related with $M_{0}$ and $M$ respectively.

In order to handle with surfaces, two important pieces of data associated with any surface are recalled: the first and second fundamental forms of surface. Let $M$ be a point on the surface whose coordinates are $\Theta^{\alpha}$ and $Q$ be a neighboring point of the surface with coordinates $\Theta^{\alpha}+d \Theta^{\alpha}$. Defining $d s$ as the elementary distance between $M$ and $Q$, the first fundamental form can be expressed by the quadratic form

$(d s)^{2}=g_{\alpha \beta} d \Theta^{\alpha} d \Theta^{\beta}$.

where the elements $g_{\alpha \beta}=\mathbf{g}_{\alpha} \cdot \mathbf{g}_{\beta}$ are the covariant components of the surface metric tensor $\mathbf{g}=$ $g_{\alpha \beta} \mathbf{g}^{\alpha} \otimes \mathbf{g}^{\beta}$. This symmetric and positive-definite tensor can be expressed on covariant, $\mathbf{g}_{\alpha}$, or contravariant, $\mathbf{g}^{\alpha}$, basis where $\mathbf{g}_{\beta} \cdot \mathbf{g}^{\alpha}=\delta_{\beta}^{\alpha}$ and $\delta_{\beta}^{\alpha}$ is the Kronecker symbol. The first fundamental form is an invariant of surface and encodes the intrinsic data about the surface without referring back to the ambient space where the surface lies.

The second fundamental form, on the other hand, is a surface invariant that encodes the information about how the surface is curved in the embedding space. From the analysis of the differential 
geometry, it follows that the covariant components of surface curvature tensor $\mathbf{b}=b_{\alpha \beta} \mathbf{g}^{\alpha} \otimes \mathbf{g}^{\beta}$, associated with the second fundamental, are given by

$b_{\alpha \beta}=\frac{\partial \mathbf{g}_{\alpha}}{\partial \Theta^{\beta}} \cdot \mathbf{g}_{3}$

where

$\mathbf{g}_{3}=\frac{\mathbf{g}_{1} \times \mathbf{g}_{2}}{\left\|\mathbf{g}_{1} \times \mathbf{g}_{2}\right\|}=\mathbf{g}^{3}$

The interest is focused in the two principal curvatures $\kappa_{I, I I}$ at a given point $M$ in the membrane surface. Considering now the eigenvalue problem in each point $M$

$\left(\mathbf{b}-\kappa_{I, I I} \mathbf{g}\right) \mathbf{v}_{I, I I}=\mathbf{0}$

where the surface shape operator is given by $\mathbf{b g}^{-1}$. Since the shape operator is self-adjoint on the tangent plane at the surface in $M$, the corresponding eigenvalues $\kappa_{I}, \kappa_{I I}$ are called the principal curvatures at $M$, and $\mathbf{v}_{I}$ and $\mathbf{v}_{I I}$ are the principal curvature directions at $M$. Recalling the definition of the Gaussian-curvature, $\mathcal{K}$, and mean-curvature, $\mathcal{H}$, it follows that

$\mathcal{K}=\operatorname{det}\left(\mathbf{b g}^{-1}\right)=\kappa_{I} \kappa_{I I}$

and

$\mathcal{H}=-\frac{1}{2} \operatorname{trace}\left(\mathbf{b g}^{-1}\right)=\frac{1}{2}\left(\kappa_{I}+\kappa_{I I}\right)$

As consequence, the maximal and minimal curvatures resulting from the quadratic form $\kappa^{2}-2 \mathcal{H} \kappa-$ $\mathcal{K}=0$ associated with the surface shape operator are given by

$\kappa_{I}, \kappa_{I I}=\mathcal{H} \pm \sqrt{\mathcal{H}^{2}-\mathcal{K}}$

For comprehensive exposition of other definitions, concepts and applications on differential geometry, see for example [30], [31] and [32].

Finally, a MatLab routine (see Appendix A) was developed to perform a two-dimensional numerical differentiation scheme over the $\Theta^{\alpha}$ coordinates for each time $t$. Thus, the terms of Eqs. (1) to (7) are computed and principal curvatures can be determined at each membrane point. Given that 3D-DIC is able to capture any 3D contour and the curvature tensor is calculated for each sur- 
face point, the presented scheme of curvature calculation is not restricted to shape characteristics like axisymmetry.

\subsection{Meridional and circumferential stress determination}

Considering now the axisymmetric bulge test, the equilibrium equations can also be derived directly by balancing the forces of deformed shape element. Employing the primary assertions of the membrane theory, every material point of the membrane in both reference and deformed configuration is in a plane stress state [33]. In Fig. 3(a) the initially flat thin planar circular sheet of radius $a$ and with an uniform thickness $h_{0}$ is presented. For convenience, to describe the strain field, cylindrical coordinates $(R, \Phi, Z=0)$ define the position of a material particle $M_{0}$ in the middle surface of the stress-free membrane in the reference configuration. Let the pressure $p$ acts in the bottom surface of specimen, the membrane experiences a deformation. The point $M_{0}$ moves to the position $M$, and the deformed configuration is defined by the coordinates $r(R, \Phi), \phi(R, \Phi)$ and $z(R, \Phi)$. Note that in either configuration, the profile of the membrane has no circumferential dependence. Under the assumption of material isotropy, the principal directions of both stretch and stress at each material particle are known ab initio to be the meridional and circumferential directions to the membrane surface. From this point forward, these directions will be denoted by the subscripts $m$ and $c$ respectively, as shown in Fig. 3(b).

Assuming quasistatic motion, the equations of equilibrium, at point $M$, for a thin axisymmetric isotropic membrane, as adopted by Hill [12], can be expressed as

$$
\begin{aligned}
\sigma_{m} & =\frac{p}{2 h \kappa_{c}} \\
\sigma_{c} & =\frac{p}{2 h \kappa_{c}}\left(2-\frac{\kappa_{m}}{\kappa_{c}}\right),
\end{aligned}
$$

where $p$ is the time-dependent normal pressure acting uniformly $(d p / d R=0)$ and $h$ the current thickness. The stress components are expressed in Eqs. 8 and 9 directly in terms of pressure and the current bulge geometry. As mentioned in [18] and [34] a remarkable consequence of membrane theory is that it admits equilibrium solutions without explicitly requiring a constitutive equation, since the equilibrium equations are derived directly by balancing forces of an element of the deformed shape. As consequence they are valid for all classes of in-plane isotropic materials. As pointed out by Hsu et al. [23], however, principal curvatures $\kappa_{m}, \kappa_{c}$ can be experimentally computed, thus Eqs. (8) 

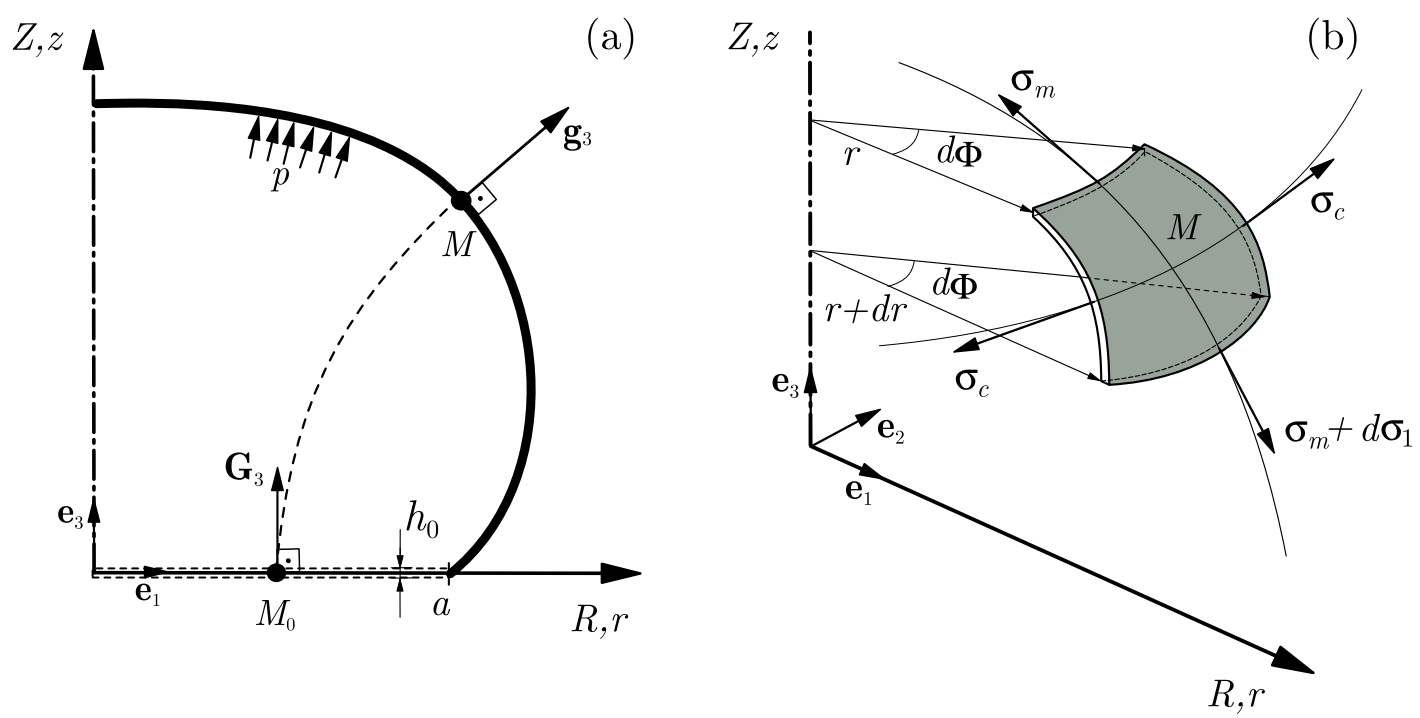

Fig. 3 (a) The axisymmetric bulge test scheme. (b) A deformed differential element and principal directions of stresses.

and (9) are very useful for exploiting axisymmetric bulge test data. Given the magnitude of error associated with the measurement system, errors in the stress determination using these equations are overwhelmingly the result of membrane theory. This point will be clarified in Section 3.3 where membrane hypotheses are investigated.

\section{Benchmark inflation test using a Mooney membrane}

\subsection{Experimental data emulation}

In order to demonstrate some quantitative predictions of the presented method to obtain the membrane stress field presented in Sec. 2 the finite element method (FEM) was used to emulate experimental data similar to that obtained by 3D-DIC. The aim here is to use a regular finite element mesh that represents the grid used by 3D-DIC to describe the surface geometry. These virtual data are obviously free from experimental problems such as missing and outlying values usually encountered in real 3D-DIC.

Using the Mooney hyperelastic model [35], the FEM outputs nodal coordinates and displacements are used as input data. An uniform mesh with an element edge length of $2 \mathrm{~mm}$ was used to discretize a square zone inscribed into the circular geometry to obtain a good spatial resolution 
for the curvatures calculation. The Mooney model was arbitrarily chosen; any other constitutive model could have been used as well. For details of Mooney model and basic equations on large deformations see [14].

\subsection{Finite element model description}

Numerical simulations were performed using the finite element code ABAQUS. Assuming an incompressible isotropic hyperelastic material behavior, the [35] strain-energy function $\mathcal{W}_{\mathrm{M}}$ can be written as

$\mathcal{W}_{\mathrm{M}}\left(I_{1}, I_{2}\right)=C_{10}\left(I_{1}-3\right)+C_{01}\left(I_{2}-3\right)$

where $C_{10}$ and $C_{01}$ are material parameters. $I_{1}$ and $I_{2}$ are the first and second strain invariants of the right Cauchy-Green deformation tensor $\mathbf{C}\left(\mathbf{C}=\mathbf{F}^{T} \mathbf{F}\right.$, where $\mathbf{F}$ is the deformation gradient tensor). Due to its mathematical simplicity as well as its prediction accuracy in the range of moderatelylarge strains, this hyperelastic model have been widely employed in the description of the behavior of rubber-like materials.

The model geometry consists in a thin circular plate, of initial radius $R(a)=90 \mathrm{~mm}$ with $h_{0}=2 \mathrm{~mm}$ of thickness. The circular plate is simply supported and subjected to an uniform inflating pressure acting perpendicular to the current configuration. The Mooney hyperelastic model parameters are those given in [14], where $C_{10}=0.14 \mathrm{MPa}$ and $C_{01}=0.024 \mathrm{MPa}$ for the RTV141 silicone rubber cured at $70^{\circ} \mathrm{C}$ during $150 \mathrm{~min}$. The finite deformation problem was modeled with the S4R shell elements (4-node doubly curved general-purpose shell, reduced integration with hourglass control, finite membrane strains) with eleven Gauss integration points through the thickness. Fig. 4(a) depicts a sliced sequence of deformed configurations for two pressure loads of $7.5 \mathrm{kPa}$ and $15 \mathrm{kPa}$, respectively.

3.3 Curvature Method: application to the virtual data

Using the FEM deformed nodal coordinates output, the principal curvatures $\kappa_{m}, \kappa_{c}$ are computed over all surface, as described in Sec. 2.3, by means of the MatLab implemented post-treatment routine, presented in Appendix A. To illustrate the curvature evaluation, the isovalues are presented in Fig. 5 for $p=15 \mathrm{kPa}$. This figure shows clearly the bulge axisymmetry, as curvatures iso-curves 

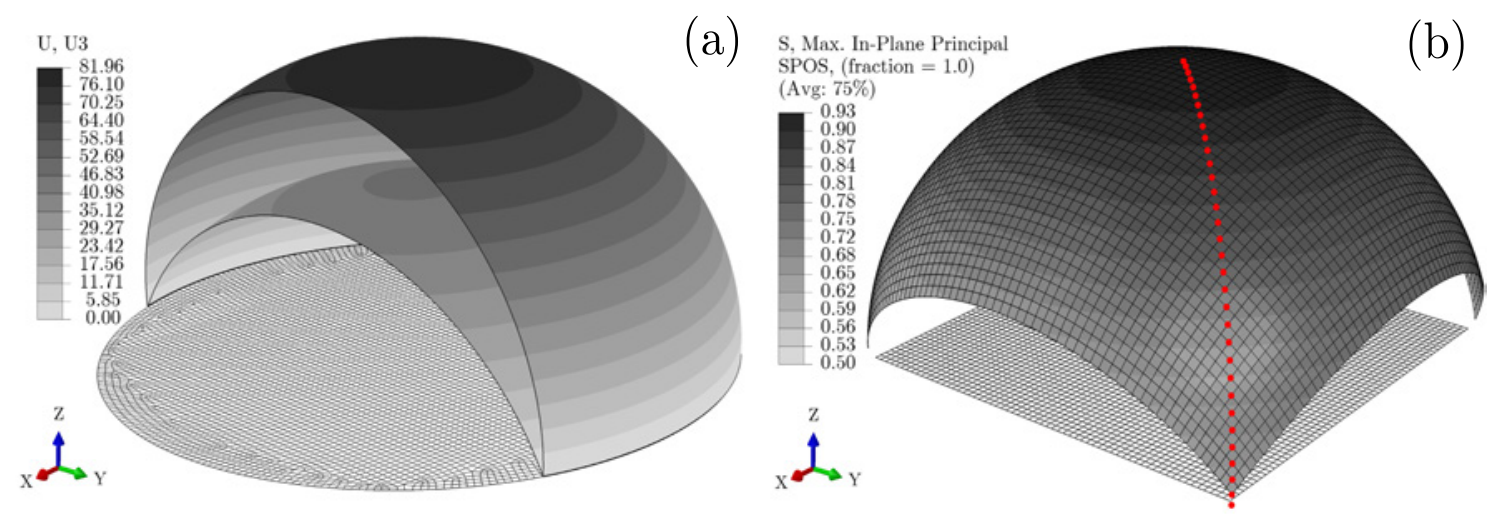

Fig. 4 Numerical inflation of Mooney behavior membrane. (a) $z$ direction displacements (in mm) for pressure loads of $7.5 \mathrm{kPa}$ and $15 \mathrm{kPa}$. Mesh are represented only in reference configuration and the two pressure steps are superposed; (b) Principal in-plane stress (in MPa) are plotted onto deformed shape and only the regular square zone is represented. Solid circles represent the meridional path.

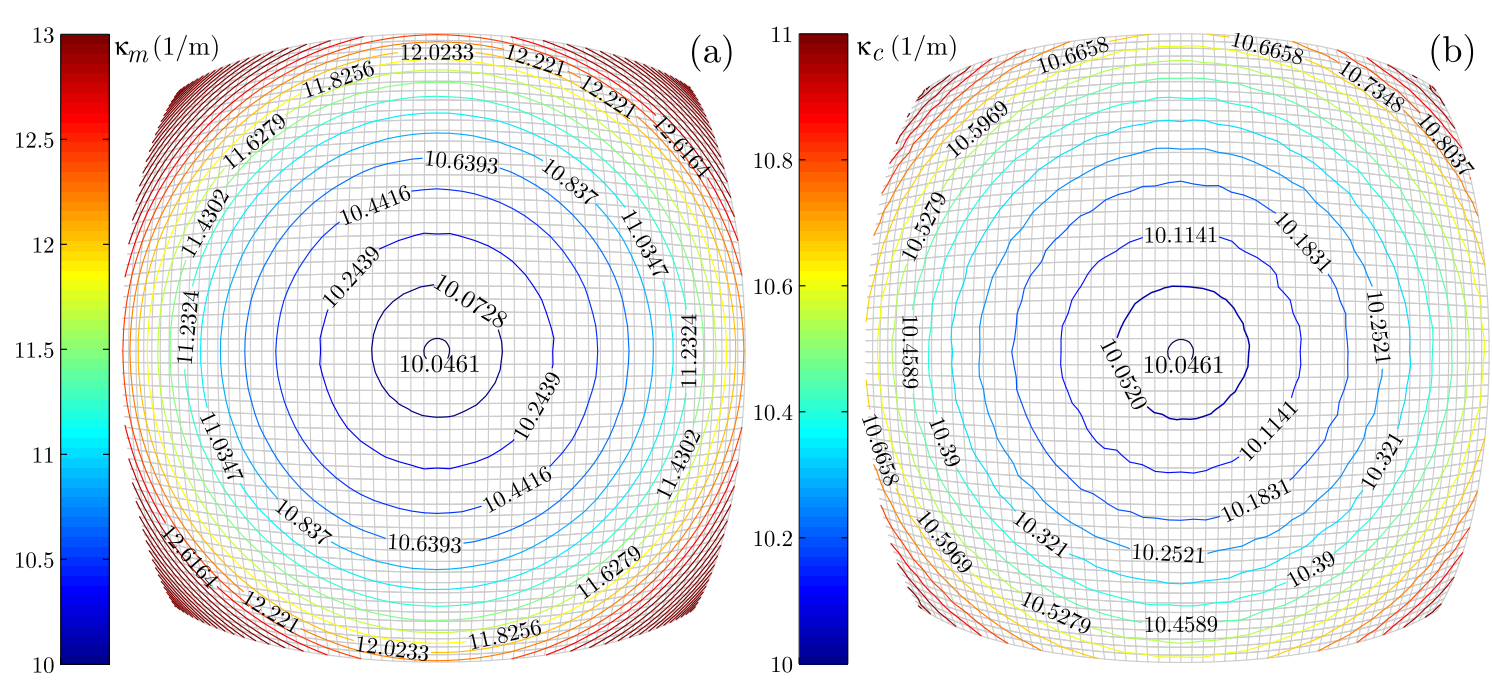

Fig. 5 Curvatures of Mooney behavior membrane at $p=15 \mathrm{kPa}$. (a) Meridional and (b) circumferential isovalues evaluated using the FEM deformed nodal coordinates output as data.

are perfectly concentric. Stress components can thus be evaluated from Eqs. (8) and (9). Moreover, since boundary conditions are perfectly balanced over the circumferential perimeter at $R(a)$, a perfectly spherical cap is obtained, i.e., surrounding the pole a cap of umbilical points $\kappa_{m}=\kappa_{c} \neq 0$ can be easily identified. At such points, both principal curvatures are equal and every tangent vector is a principal direction.

The output fields principal stretches $\left(\lambda_{m}, \lambda_{c}\right)$, principal curvatures $\left(\kappa_{m}, \kappa_{c}\right)$ and principal stresses $\left(\sigma_{m}, \sigma_{c}\right)$ from the FEM are plotted for a single meridional path in Fig. 6(a), (b) and (c), respectively. 
Afterward, the principal stresses $\left(\sigma_{m}, \sigma_{c}\right)$ computed using Eqs. (8) and (9) were compared with the Mooney membrane FEM outputs. It can be seen that the results fit very well in Fig. 6(c). The relative differences between both stress components using the presented method and FEM are plotted in Fig. 6(d) for $7.5 \mathrm{kPa}$ and $15 \mathrm{kPa}$. Errors tend to be less than $2 \%$ for both stress directions for maximum pressure level of $15 \mathrm{kPa}$. On the other hand, errors increase for lower pressure levels. This result is natural knowing that principal curvatures vanish when the surface becomes flat. However, the principal explanation in disagreement of the stress results comes from the fact that, no matter how thin, all structures are three-dimensional and possess some bending stiffness. It is important to bear in mind that the membrane theory is an approximation. Fig. 7 shows the variation of FEM principal stresses over the membrane thickness $h$ in the pole $(R=0)$ and in the border $(R=0.95 a)$, for an initial ratio radius/thickness of $a / h=45$. Note that, bending effects are more pronounced nearest to the border $(R \rightarrow a)$ in both configurations. This fact justifies the form of curves presented in Fig. 6(d). The other important observation from Fig. 7 is that the bending influence can increase for low pressure levels. A similar result was found by Kyriacou et al. [36] for an inverse finite element characterization using a bulge test sample with $a / h \approx 30$ stretched at the pole of about 1.3. The authors noticed that the error in the estimated parameter increased with decrease of the pressure at which the data were generated. Depending on the material and geometry of the sample, the amount of bending could be non-negligible at small strain levels.

A last important remark arises from the fact that curvatures estimated for real 3D-DIC data are very sensitive to experimental noise since estimates of partial derivatives of second order are necessary. Gaussian noise was then introduced into the $X^{i}$ coordinates of FEM data results to simulate potential experimental errors. Applying our method to these noisy data leads to very poor estimation of both circumferential and meridional stresses, as shown in Fig. 8. Smoothing can be used to reduce experimental additive noise and small-scale information while keeping the most important imprints of data set. For these cases, a discretized smoothing spline algorithm proposed by Garcia [37] was implemented. The weighted robust version of the algorithm was used to deal with occurrences of missing and outlying values of gridded data. The perturbed data were used as input of post-treatment routines. Curvatures and stresses were recalculated and Fig. 8 presents the results. Smoothing allows to obtain very close estimated values of the stresses from the noisy data to those of the original FEM data. 


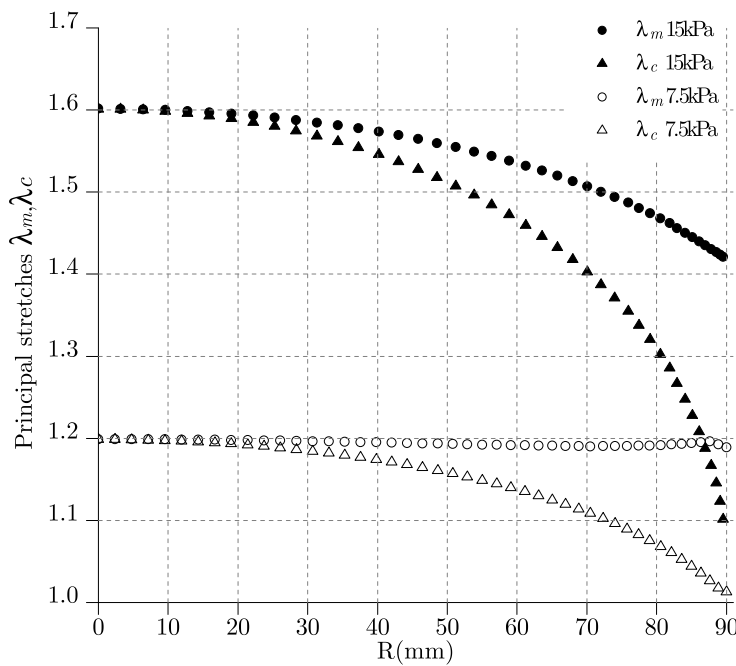

(a)

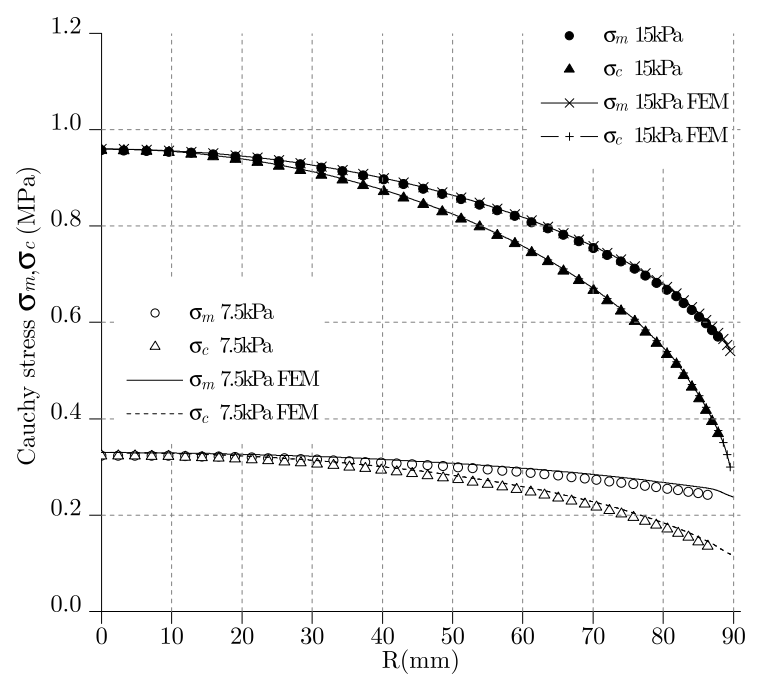

(c)

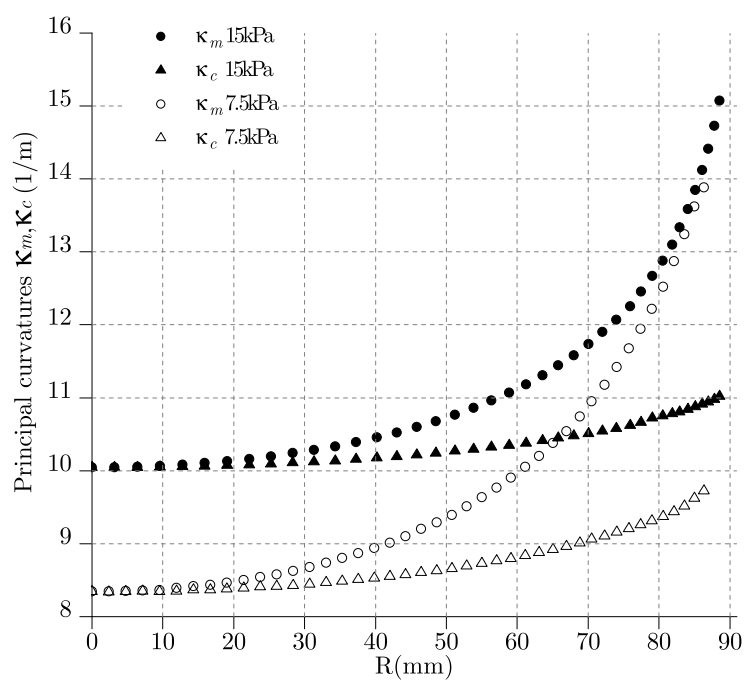

(b)

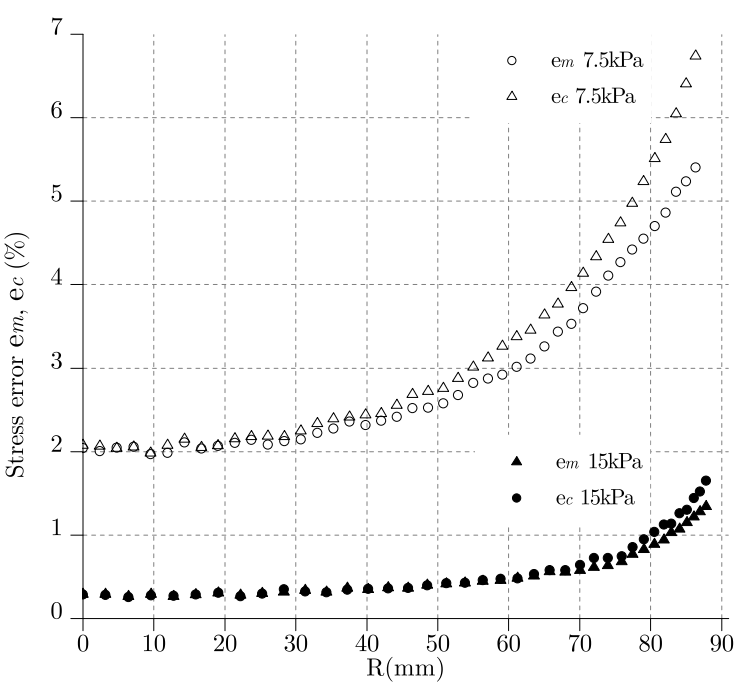

(d)

Fig. 6 Two deformed configurations $7.5 \mathrm{kPa}$ and $15 \mathrm{kPa}$ pressure levels for the incompressible Mooney $\left(C_{10}=\right.$ $0.14 \mathrm{MPa}$ and $\left.C_{01}=0.024 \mathrm{MPa}\right)$ membrane. (a) Principal stretches $\left(\lambda_{m}, \lambda_{c}\right)$; (b) Principal curvatures $\left(\kappa_{m}, \kappa_{c}\right)$; (c) Cauchy stress $\left(\sigma_{m}, \sigma_{c}\right)$ confronted with the finite element results (FEM); (d) Errors with respect to principal stresses in both deformed configurations.

This numerical study validates the developed post-treatment routines, as the stress results are quite similar to FEM results by considering the hypotheses presented. 


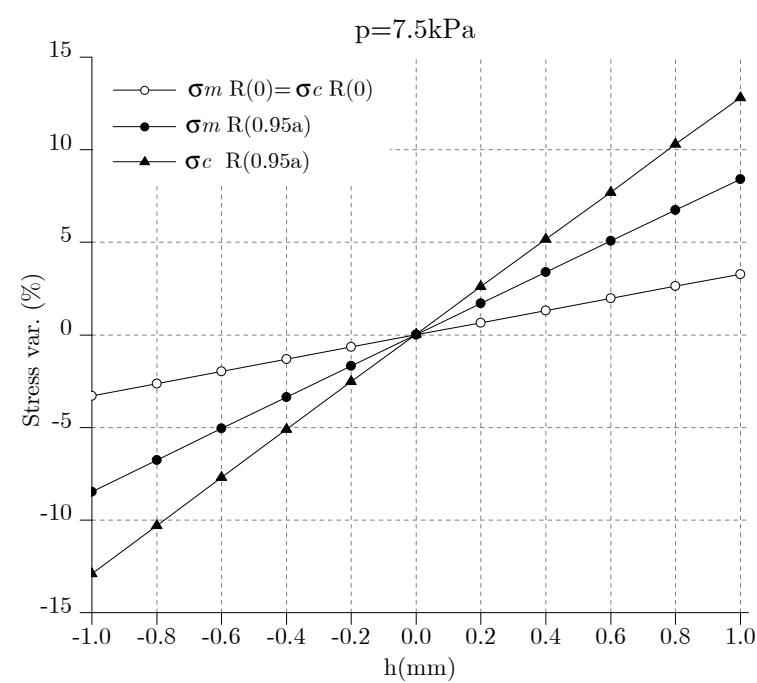

(a)

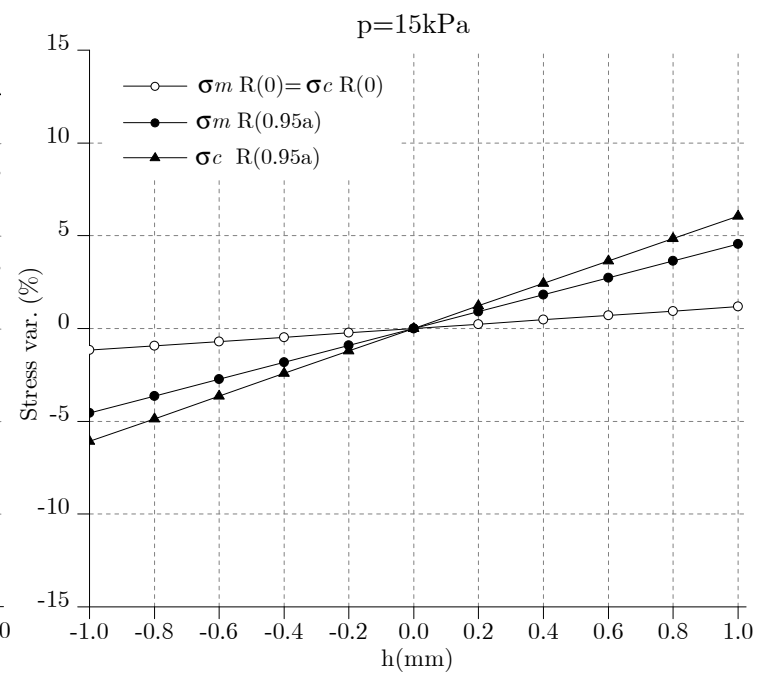

(b)

Fig. 7 Bending stiffness effects in both deformed configurations $(7.5 \mathrm{kPa}$ and $15 \mathrm{kPa}$ pressure levels) for the numerical benchmark. Principal stresses variation (i.e. $\sigma_{m} / \sigma_{m(m e a n)}$ analogous for $\sigma_{c}$ ) over the element thickness $(h)$ at the sample pole $(R(0)=0 \mathrm{~mm})$ and border $(R(a)=90 \mathrm{~mm})$.

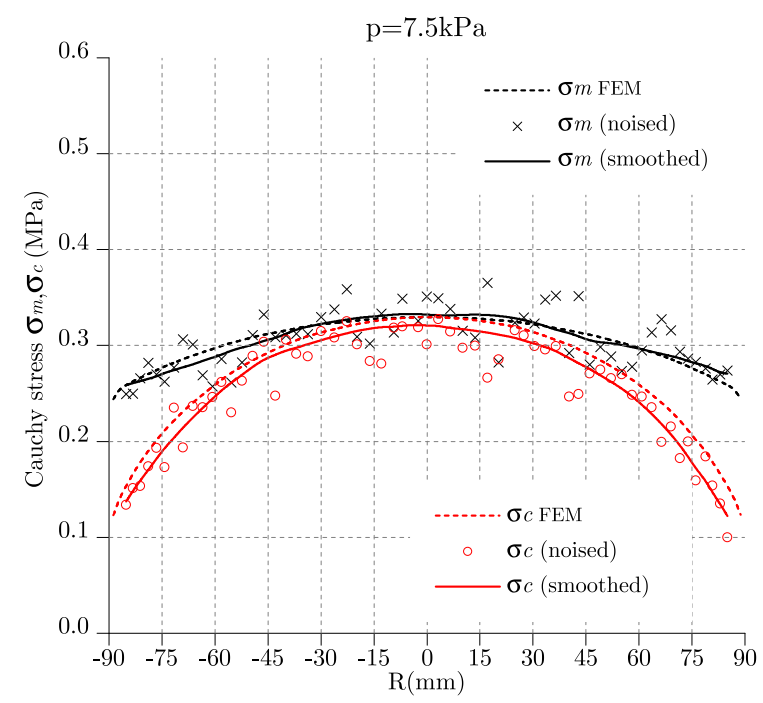

(a)

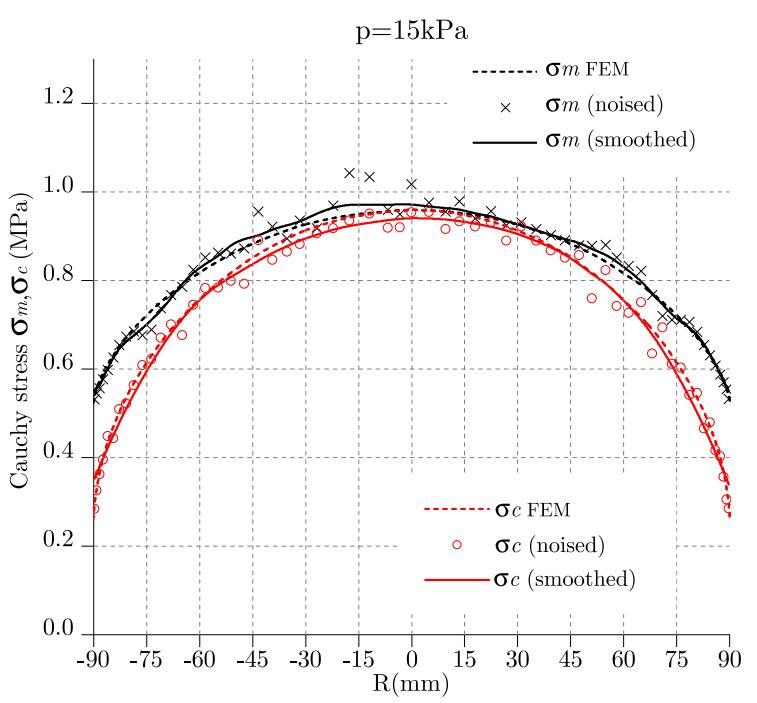

(b)

Fig. 8 Principal stress $\left(\sigma_{m}, \sigma_{c}\right)$ results. Original finite element output (FEM), calculated using the artificially noised data (noised) and calculated using the smoothed data (smoothed).

\section{Application of the method to experimental bulge test of a silicone elastomer}

\subsection{Experimental bulge test}

The objective of this section is to experimentally validate the proposed method to determine curvatures and stresses during a bulge test of a silicone elastomer. Experimental difficulties are 
pointed out and results are confronted with a finite element model. The chosen silicone rubber is an unfilled formulation produced by Rhodia (RTV 141). Specimens were produced using the processing described in [14], following the processing route: (i) mixing the two liquid components (uncured silicone and curing agent with a 10/1 ratio), (ii) putting the uncured mixture under vacuum for $30 \mathrm{~min}$ in order to eliminate undesirable entrapped bubbles, (iii) injecting the liquid mixture in sheet mold and (iv) putting mold inside an oven at $70{ }^{\circ} \mathrm{C}$ for 4 hours to cure the silicone. Compared to [14], the cure was augmented in order to increase the tear resistance of the material. This allows to largely swell the membranes. Using the RTV141 silicone, experimental bulge tests were carried out to determine a non-uniform stress strain state over the membrane surface.

\subsubsection{Bulge test setup}

The specimen disk dimensions are the same as in Sec. 3 (initial radius $R(a)=90 \mathrm{~mm}$; thickness $h_{0}=2 \mathrm{~mm}$ ) constrained between two clamping flanges. In the used test machine, a pressurized air line is connected to the bottom circumferential clamp. Given the small pressures used during the experiments, a regulator is required to reduce the line pressure to a maximum of $30 \mathrm{kPa}$. To decrease the pressure an unloading valve is opened to create a leak. The internal pressure is measured by a Foxboro $0-500$ mbar pressure sensor. A picture of the experimental setup is shown in Fig. 9(a).

\subsubsection{D-DIC setup and experimental difficulties}

The Dantec Q-400 commercial 3D-DIC system was used to acquire and correlate the full-field bulge shape information. Since the silicone sample experiences a large out-plane displacement, an appropriate focal length setup is absolutely necessary. The CCD cameras, using $20 \mathrm{~mm}$ focal length lenses opened at $f / 16$, are arranged so that the pole and border of the circular membrane are present in both camera image planes. It is important that the object surface shows enough structure to allow the algorithms to correlate identical points from both cameras. Note that, using only two cameras, information near to the border will be lost if the membrane experiences large displacements creating a self-shadow effect in this region. A parameter $R_{\max }$ has been used to define the limit of the correlation zone, i.e., the maximum distance from the membrane center which is seen by the two cameras. A third camera could be added in this case to be able to correlate along all meridional direction, see [38] for a multiple-camera instrumentation example. 
High spatial resolution is needed to allow an accurate measurement of curvatures. The spatial resolution achieved in practice depends on a number of factors, including but not limited to camera resolution, lens optical quality, and marker size and quality. Facet size also limits the spatial resolution strains since the greyscale information within a facet is used for the correlation between images. An increase of the facet size causes smoothing of the results, decreasing the spatial resolution. In order to obtain independent data points, the facets of neighboring data points should not overlap. In this work, full camera resolution $\left(C_{r e s}\right)$ is used $(2048$ pixels $\times 2048$ pixels $)$ with an evaluation grid size $G_{s}=12$ pixels and a facet size $f_{\text {size }}=21$ pixels, resulting in a spatial resolution of $S_{r}=1.45 \mathrm{~mm}$ over the membrane surface.

Fig. 9(b) presents an example of reconstructed surface. Note that some data are missing in some small regions on surface. It occurs due to some correlation abnormalities, normally associated with locally irregular pattern.

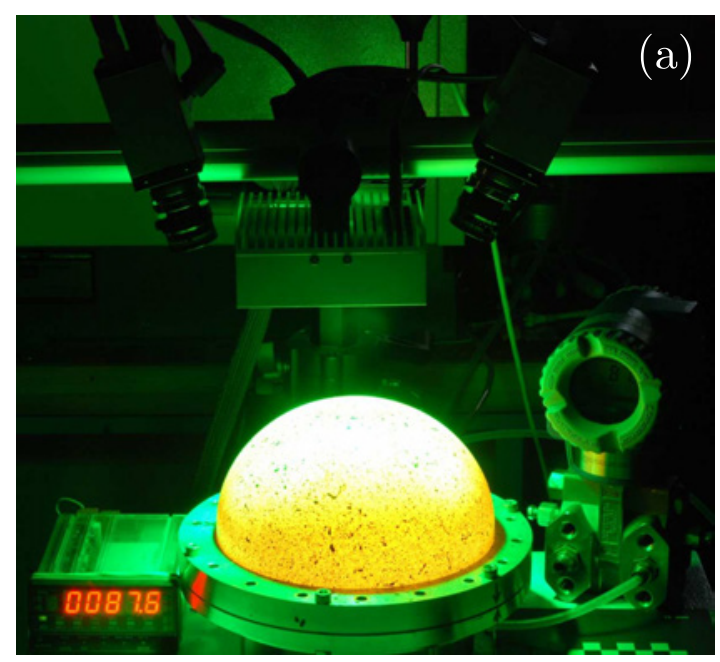

Fig. 9 (a) The bulge test experimental setup using 3D-DIC technique; surface.

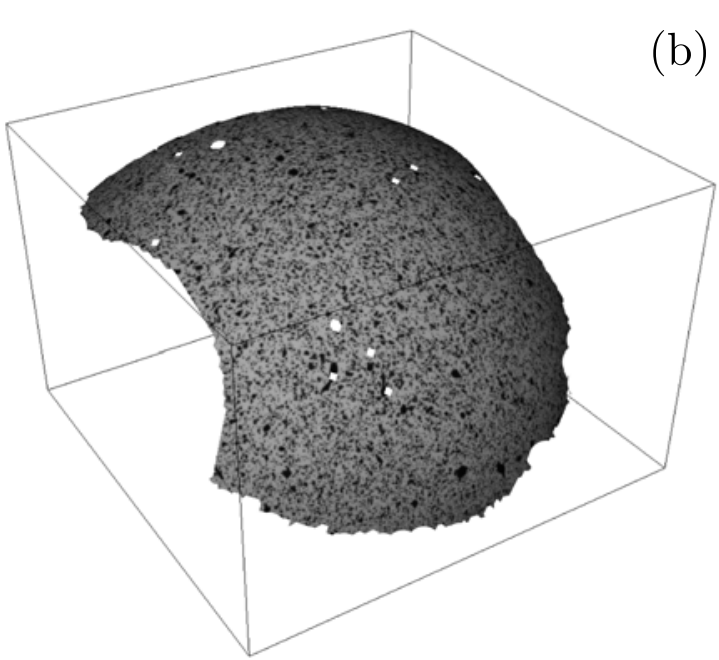

(b) Reconstructed RTV141 membrane (b)

\subsubsection{Test Results}

Cyclic inflations were performed, applying $p=30 \mathrm{kPa}$ several times, to pre-stretch the specimen in order to accommodate the material. This is due to the fact that silicone rubber can exhibit considerable stress softening phenomenon for different loading cases [39]. After a recovery time of 


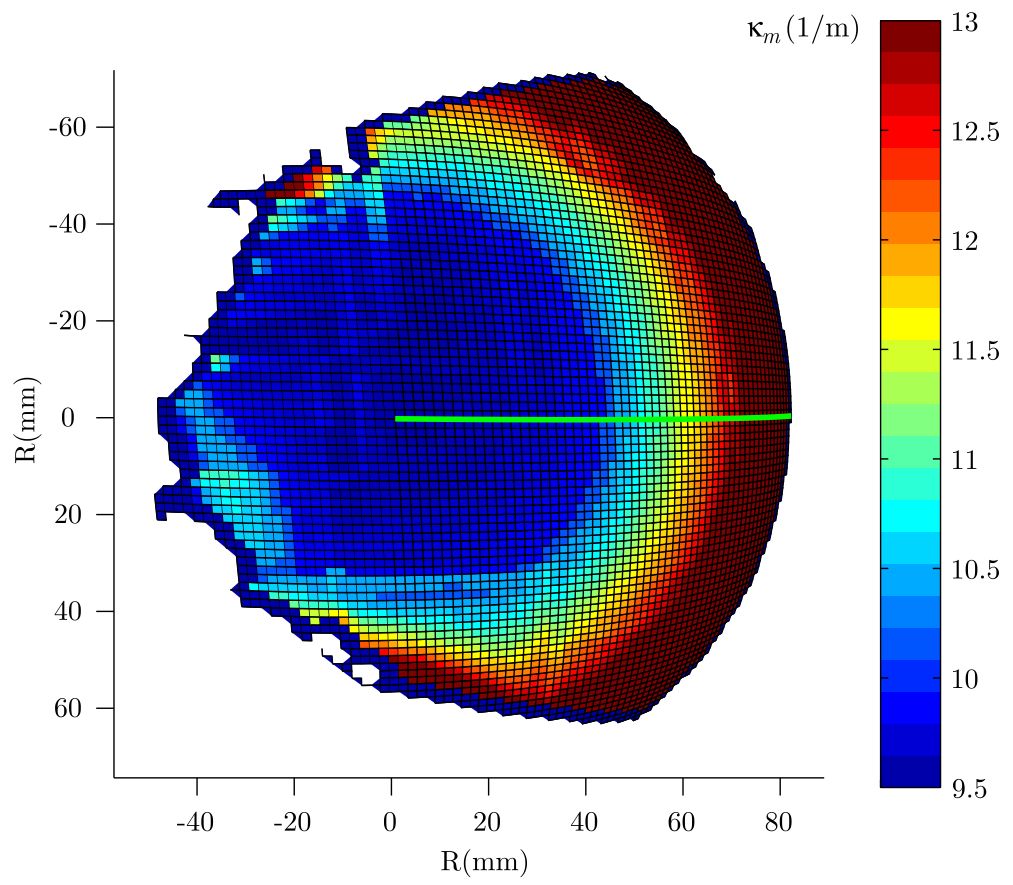

Fig. 10 Principal curvature $\kappa_{m}$ plotted onto 3D-DIC reconstructed surface. Solid line represents the meridional path used in Fig. 11

$1 \mathrm{~h}$ and starting from an initially flat surface, inflations were performed from $8 \mathrm{kPa}$ to a maximum pressure of $27.1 \mathrm{kPa}$. These inflations yielded principal stretches at the pole of about 1.10 and 1.36 respectively. Fig. 10 shows the principal curvature $\kappa_{m}$ plotted onto 3D-DIC reconstructed surface. This bulge test can be considered as axisymmetric. Plots of the deformed membrane, over the meridional path, for each of the two inflation states can be found in Fig. 11. The values of $z$ direction displacements (in mm) and principal stretches $\left(\lambda_{m}, \lambda_{c}\right)$ were obtained direct from 3D-DIC system. Principal curvatures $\left(\kappa_{m}, \kappa_{c}\right)$ and principal stresses $\left(\sigma_{m}, \sigma_{c}\right)$ were computed using Eqs. (7), (8) and (9) respectively.

For each of the two inflation states all material points, save the pole $(R(0)=0 \mathrm{~mm})$ and the clamped boundary $(R(a)=90 \mathrm{~mm})$, experience both axial and meridional displacements as a result of their respective finite deformations. The plots of the principal stretches $\left(\lambda_{m}, \lambda_{c}\right)$ in Fig. 11(c) show that each inflation state involves a heterogeneous strain state, i.e., equibiaxial stretch at the pole, planar stretching at $R(a)$. As expected, the circumferential stretch $\lambda_{c}$ tends to one towards the clamped boundary, i.e., when $R \rightarrow R(a)$. With respect to principal curvature distributions $\left(\kappa_{m}, \kappa_{c}\right)$, note that equibiaxial membrane deformations near the membrane pole are associated with an 


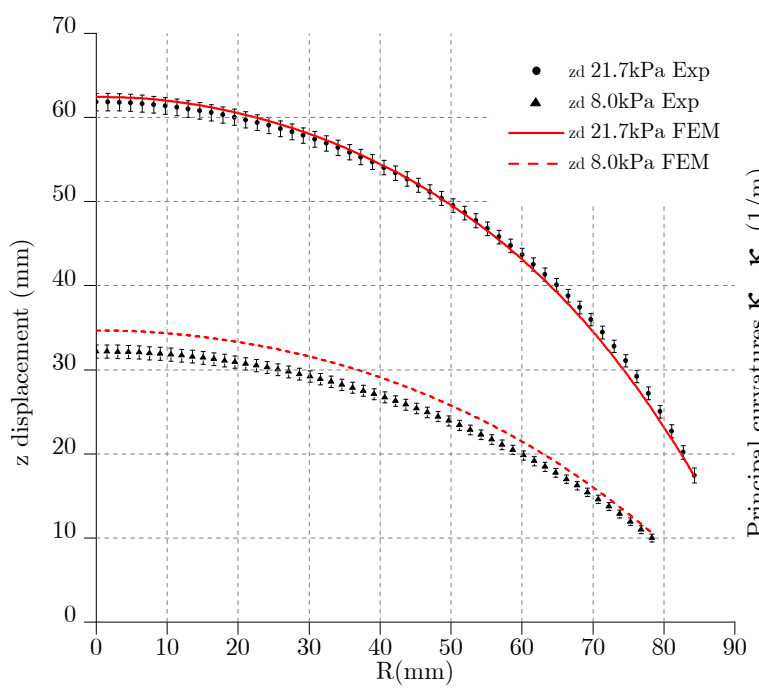

(a)

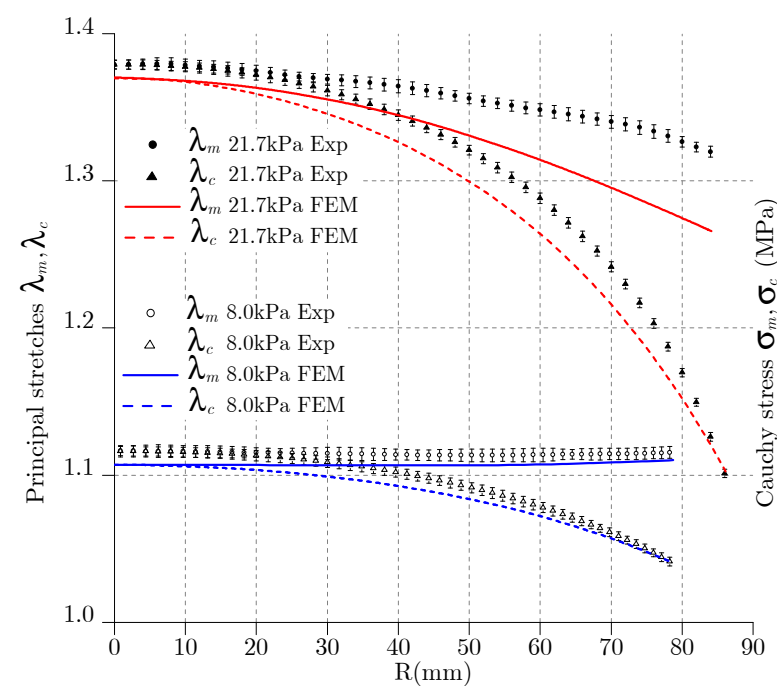

(c)

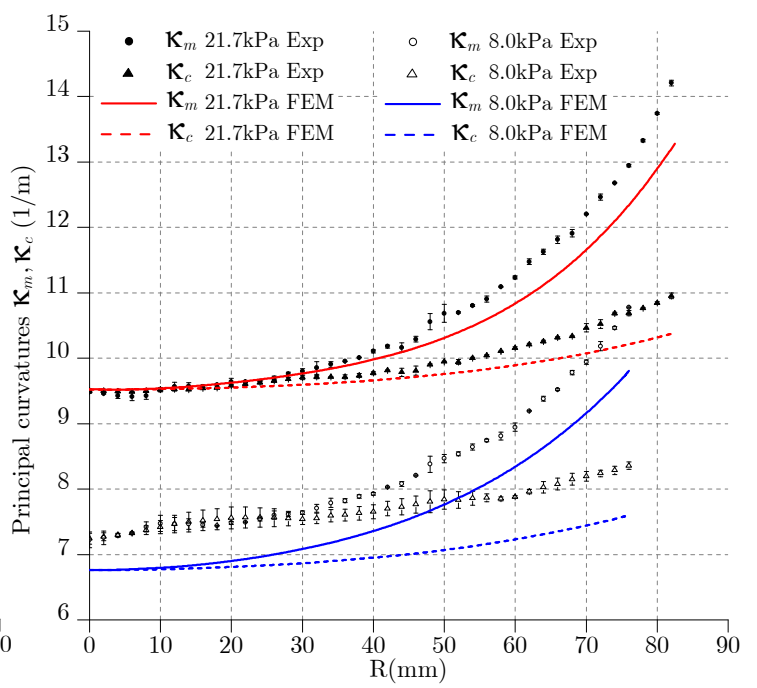

(b)

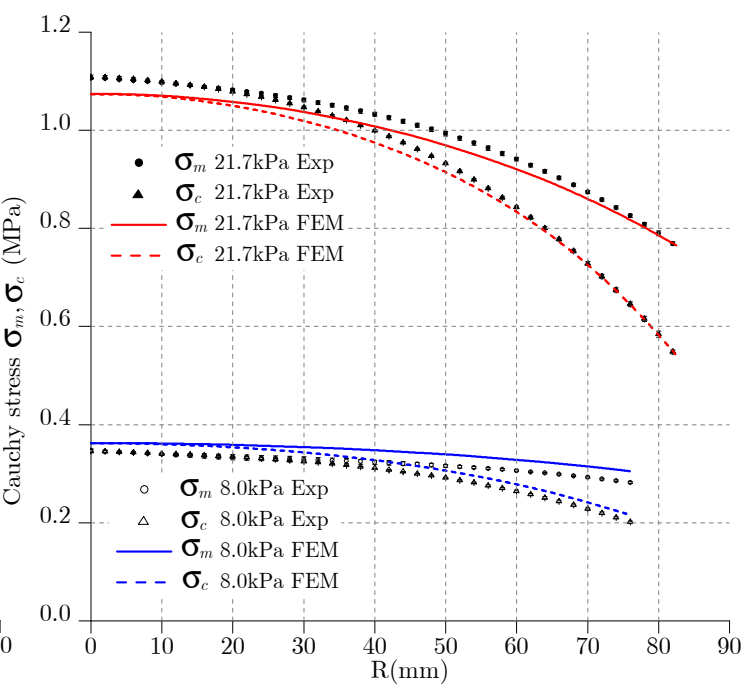

(d)

Fig. 11 Distributions of experimental (Exp) and numerical (FEM) fields: (a) $z$ direction displacements; (b) principal curvatures $\left(\kappa_{m}, \kappa_{c}\right)$; (c) principal stretches $\left(\lambda_{m}, \lambda_{c}\right)$; (d) principal stresses $\left(\sigma_{m}, \sigma_{c}\right)$; corresponding to $8 \mathrm{kPa}$ and $21.7 \mathrm{kPa}$ inflation states.

approximately spherical geometry, i.e., $\kappa_{m} \approx \kappa_{c}$. The non perfect equality may be explained by the fact that the umbilical point may not lie exactly on the $z$ direction axis. This subtile difference can increase for high deformed states. Stress state can be assumed to be approximately balanced-biaxial at the pole, following by the circumferential stress $\sigma_{c}$ which steadily decreases when $R \rightarrow R(a)$. 
4.2 Material characterization and numerical simulation

In the previous section, stress and strain fields have been obtained by the presented method which does not require any constitutive equation and parameter determination. The aim of this section is to compare these results with usual analysis of bulge tests. This analysis is done thanks to a numerical simulation implying a modeling of the mechanical behavior of the unfilled silicone rubber RTV141. It is known that different modes of deformation are required to obtain the constitutive equation and the parameters that define the stress-strain relationship accurately. The tests used in this investigation are cyclic uniaxial and equibiaxial tension, completely independent, i.e., they were performed in parallel to the bulge test described in the last section.

Uniaxial tensile test and equibiaxial test are performed at room temperature at $\dot{\lambda}=1.25 \times$ $10^{-2} \mathrm{~s}^{-1}$. The material presents a weak hysteresis and a very little strain rate dependency [14]. Nevertheless, a stress softening phenomenon, called Mullins effect [40] appears, contrary to [14] using a smaller curing time. Mullins effect strongly depends on the maximum previous deformation. To measure the influence of the previous maximum deformation, increasing cyclic uniaxial tests were performed at $\lambda=1.75$ and $\lambda=1.95$. Fig. 12(a) highlights that for moderate deformations $\lambda<1.40$, the second loading curves are nearly the same whatever is the previous maximum strain. This means that increasing the maximum strain has only a slight effect on the behavior at moderate deformations. Therefore, for the range of deformation, a hyperelastic constitutive equation is sufficiently accurate to model the mechanical behavior. The neo-Hookean constitutive equation [41] is chosen to describe mild deformations:

$\mathcal{W}_{\mathrm{N}}\left(I_{1}\right)=C_{10}\left(I_{1}-3\right)$

The material parameter is fitted on second loading curves for uniaxial and equibiaxial tests, $C_{10}=$ $0.335 \mathrm{MPa}$ is obtained. The results are presented in Fig. 12(a) and (b). This shows that the model predicts satisfactorily the two loading stress for $\lambda<1.40$.

4.3 Experimental and numerical comparison of bulge test results

Results of FEM using the neo-Hookean model are superposed with the experimental fields (displacement, principal curvatures, principal stretches and principal stresses) in Fig. 11. All numerical predictions clearly follow the trends calculated from the presented method to obtain the princi- 


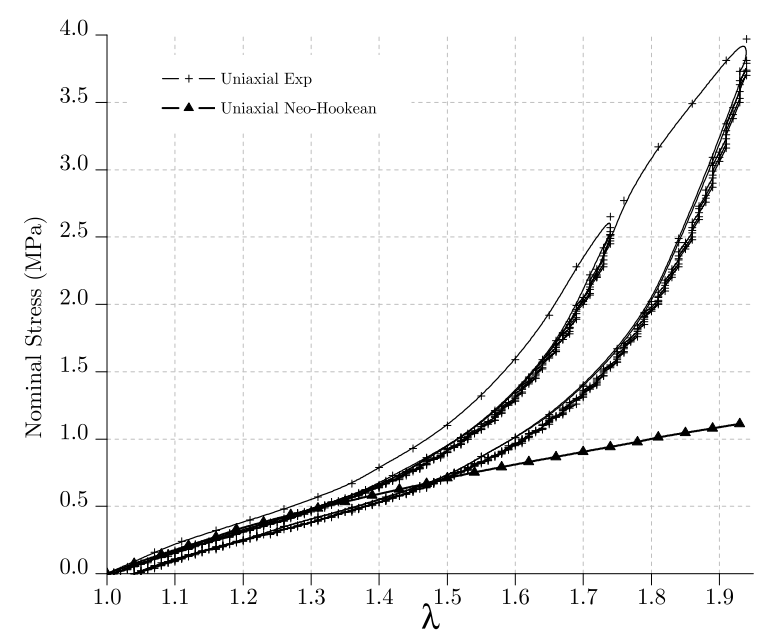

(a)

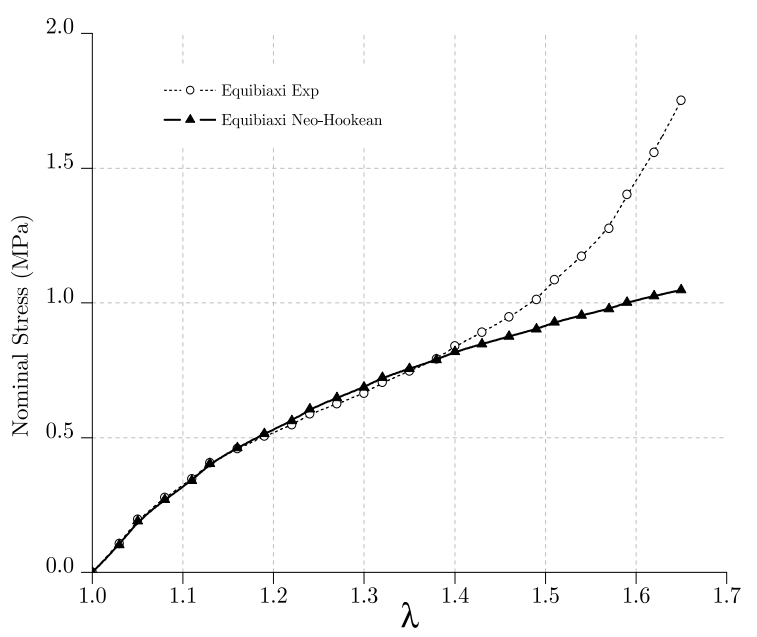

(b)

Fig. 12 Experimental data and the hyperelastic fitting using the neo-Hookean hyperelastic model: (a) Uniaxial and (b) equibiaxial tensile tests at $\dot{\lambda}=1.25 \times 10^{-2} \mathrm{~s}^{-1}$.

pal curvatures applied to the experimental data. For the $z$ displacement field (Fig. 11(a)) a pronounced difference, for the lower pressure value, is observed. Consequently the curvatures evaluation (Fig. 11(b)) for this same load state is obviously affected. Nevertheless, errors in curvatures determination decreased with an increase in the strain level as observed in the benchmark of Section 3. Considering the principal stresses plots, a good agreement is observed in spite of the hyperelastic constitutive equation simplicity.

It is important to keep in mind the simplicity of the neo-Hookean model, the simple procedure used for material parameter identification and the precaution taken to accommodate the material before testing. An interesting characterization method for rubber is proposed in [42], performing only one heterogeneous mechanical test, measuring the displacement and strain fields using DIC and applying an inverse method. In such experiment, a large set of heterogenous states was obtained and results are represented in the $I_{1} \times I_{2}$ invariants plane, between uniaxial tensile and planar shear tensile paths. Fig. 13 illustrates the strain states, in the $I_{1} \times I_{2}$ invariants plane, for the classical three homogeneous tests used in material parameter identification. In the same figure, the gray zone represents all the heterogenous states covered in the bulge test, with the advantage of knowledge about the stress field. Note that, for small values of $I_{1}$ and $I_{2}$, bending effects could be non-negligible and membrane theory fails as explained in Section 3.3. However, there is no simple rule to estimate these bending effects independently of the tested material and of the geometry of 
the bulge sample. Given a material, an estimation of the magnitude of bending effects could be done experimentally by varying the geometry of bulge sample.

For the presented material test, the distribution of loading cases in this zone are limited to $R_{\max }$ achieved during the correlation step. This zone is represented by a circular hatch pattern in Fig. 13. As aforementioned, the $R_{\max }$ depends on the membrane displacements levels and camera dispositions in order to acquire the information over the border, i.e., the zone dominated by planar shear tensile state.

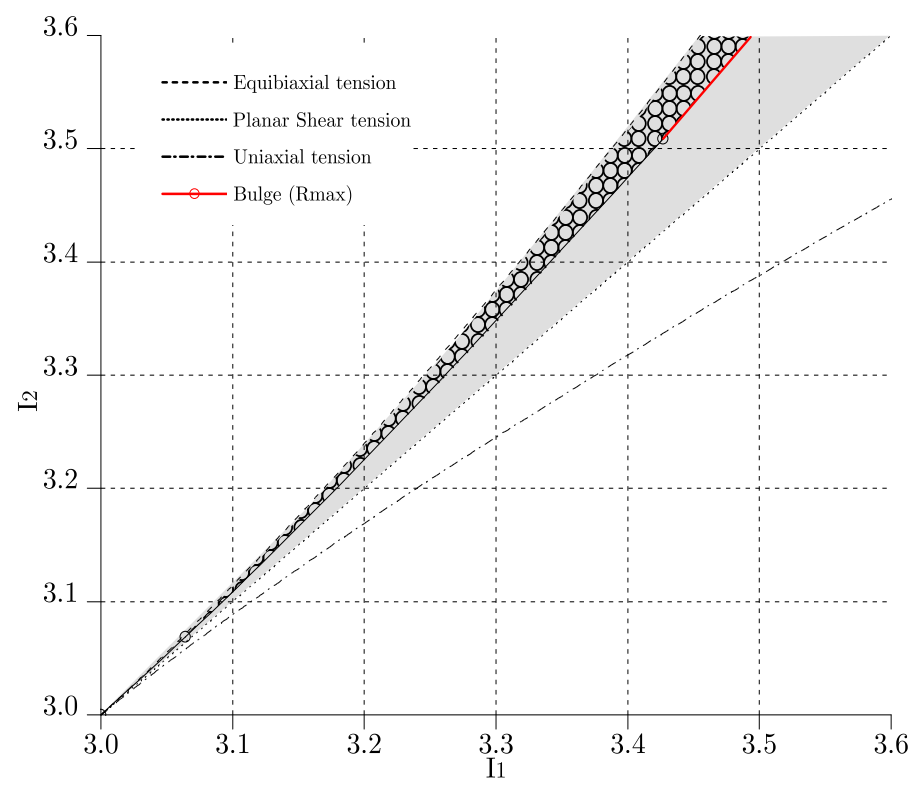

Fig. 13 Loading case in $I_{1} \times I_{2}$ plane. The gray zone represents all heterogeneous deformations states covered by the bulge test. The Bulge $\left(R_{\max }\right)$ curve refers to the heterogeneous path at $R_{\max }$ point. The circular hatch pattern represents the distribution of loading cases for the presented material test.

\section{Conclusion}

The aim of the present work was to propose a method which enriches greatly the analysis of usual bulge tests by using the 3D-DIC technique. Firstly, this paper presented a general method to determine surface curvature tensors from 3D-DIC data. This requires no assumptions regarding axisymmetry or other shape characteristics of the membrane surface. Secondly, the determination of the curvature tensor fields allowed to check the axisymmetry of the bulge test, which had to be obtained for planar-isotropic material and perfect boundary conditions. Once this axisymmetry 
was proved, the proposed method allowed to determine the stress-strain fields associated with the membrane inflation problem without any constitutive equation. The method was applied in this paper both on virtual data generated by numerically simulating the bulge test and on real data of one experiment performed on a silicon elastomer. In this last case, experimental difficulties were pointed out, particularly the difficulty to obtain the information near to the border for the chosen material. These two examples showed that bulge test can be used as a very rich heterogeneous mechanical test which allows to obtain valuable information and can be used to different types of materials. A wide range of stress-strain states, ranging from equibiaxial to planar tensile stress state, can potentially be achieved improving the experimental setup to obtain the stress-strain fields at the border for very large displacements.

Acknowledgements We would like to thank the French ANR for supporting this work through the project RAAMO ("Robot Anguille Autonome pour Milieux Opaques")

\section{Appendix A}

MatLab routine used to evaluate the surface curvatures.

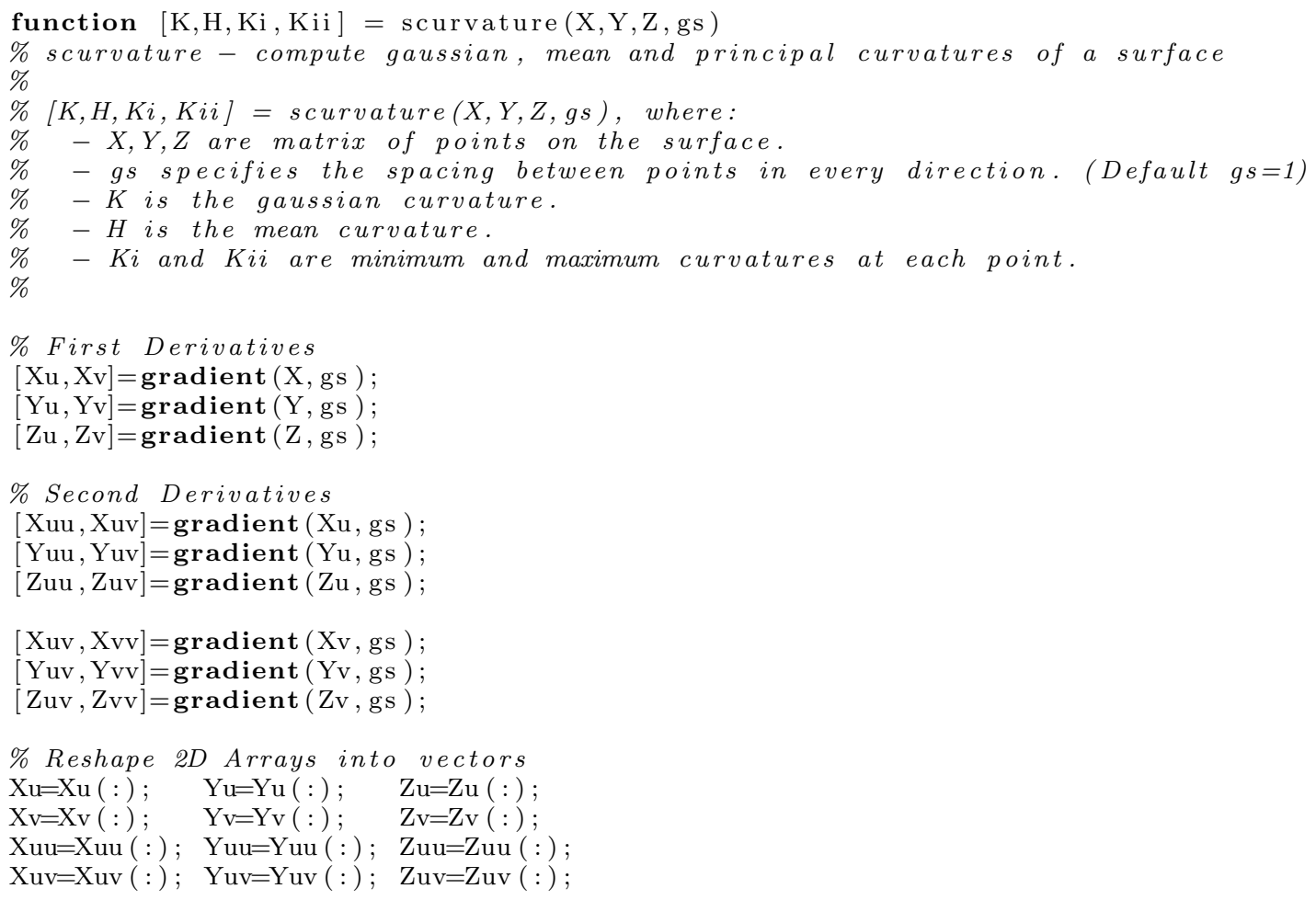




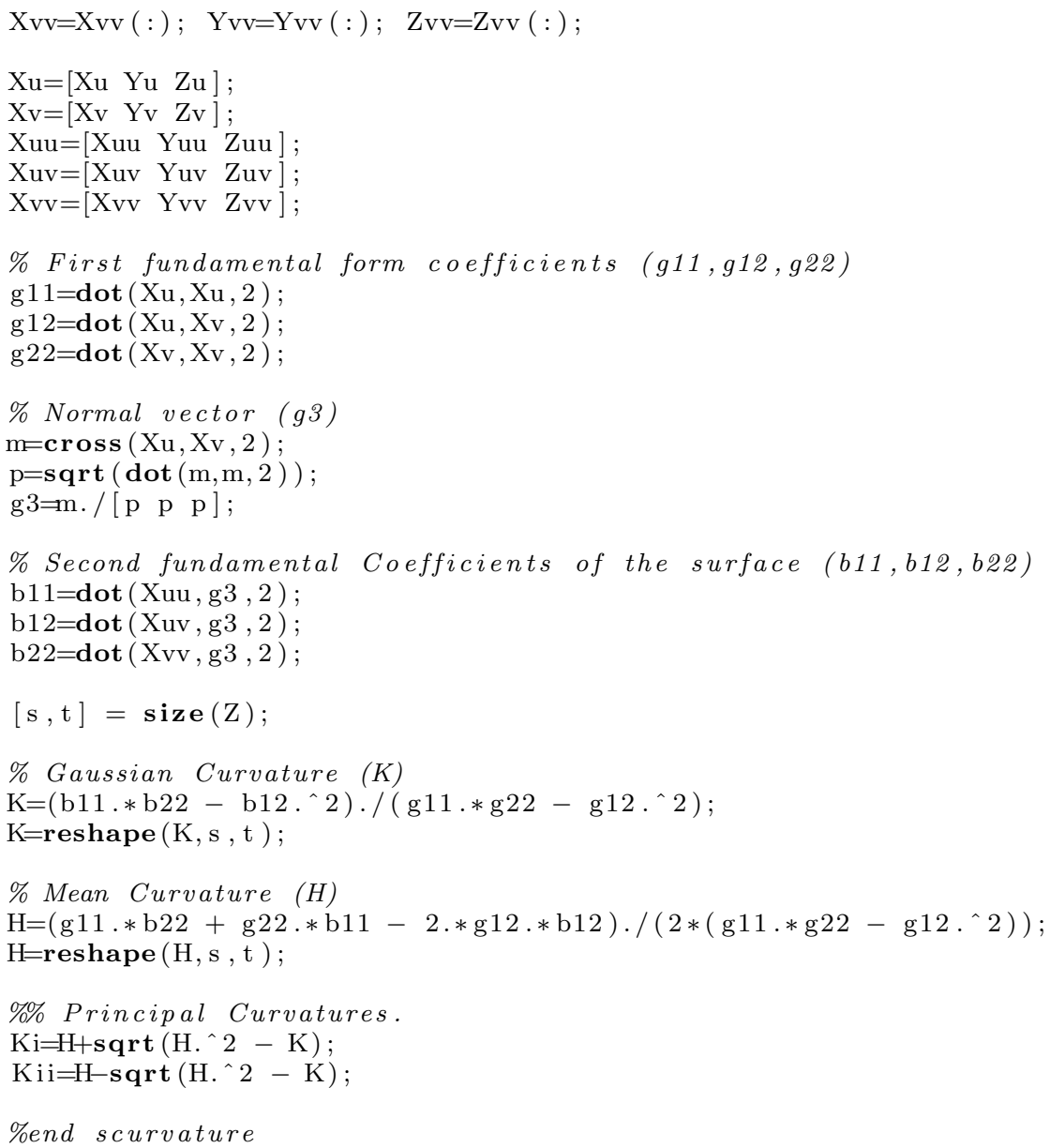

\section{References}

1. Avril, S., Bonnet, M., Bretelle, A., Grediac, M., Hild, F., Ienny, P., Latourte, F., Lemosse, D., Pagano, S., Pagnacco, E., et al. (2008). Overview of identification methods of mechanical parameters based on full-field measurements. Exp. Mech., 48(4), 381-402.

2. Treloar, L. R. G. (1944). Strains in an inflated rubber sheet and the mechanism of bursting. Trans. of the Institution of Rubber Industry, 19, 201-212.

3. Brown, W. F. and Thompson, F. (1949). Strength and failure characteristics of metal membranes in circular bulging. Trans. Am. Soc. of Mech. Engrs., 71, 575-585.

4. Tsakalakos, T. (1981). The bulge test - A comparison of theory and experiment for isotropic and anisotropic films. Thin Solid Films, 75, 293-305. 
5. Mitchell, J. S., Zorman, C. A., Kicher, T., Roy, S., and Mehregany, M. (2003). Examination of bulge test for determining residual stress, Young's modulus, and Poisson's ratio of 3C-SiC thin films. J. Aerospace Eng., 16(2), 46-54.

6. Seshaiyer, P., Hsu, F. P. K., Shah, A. D., Kyriacou, S. K., and Humphrey, J. D. (2001). Multiaxial mechanical behavior of human saccular aneurysms. Comput. Methods Biomech. Biomed. Eng., 4(3), 281-289.

7. Miller, C. E. (1979). Determination of elastic parameters for human fetal membranes. J. Rheol., 23, 57-78.

8. Kriewall, T., Akkas, N., Bylski, D., Melvin, J., and Work, B. (1983). Mechanical-behavior of fetal dura mater under large axisymmetric inflation. J. Biomech. Eng. - T. ASME, 105(23), 71-76.

9. Selby, J. C. and Shannon, M. A. (2007). Apparatus for measuring the finite load-deformation behavior of a sheet of epithelial cells cultured on a mesoscopic freestanding elastomer membrane. Rev. Sci. Instrum., 78(9), 094301.

10. Grolleau, V., Gary, G., and Mohr, D. (2008). Biaxial testing of sheet materials at high strain rates using viscoelastic bars. Exp. Mech., 48, 293-306.

11. Adkins, J. E. and Rivlin, R. S. (1952). Large elastic deformation of isotropic materials. IX. The deformation of thin shells. Phil. Trans. R. Soc., A244, 505-532.

12. Hill, R. (1950). A theory of the plastic bulging of a metal diaphragm by lateral pressure. Phil. Mag., 41, 1133-1142.

13. Ross, E. and Prager, W. (1954). On the theory of the bulge test. Q Appl. Math., 12, 8691.

14. Meunier, L., Chagnon, G., Favier, D., Orgéas, L., and Vacher, P. (2008). Mechanical experimental characterisation and numerical modelling of an unfilled silicone rubber. Polym. Test., 27, $765-777$.

15. Sasso, M., Palmieri, G., Chiappini, G., and Amodio, D. (2008). Characterization of hyperelastic rubber-like materials by biaxial and uniaxial stretching tests based on optical methods. Polym. Test., 27, 995-1004.

16. Grolleau, V., Louche, H., Delobelle, V., Penin, A., Rio, G., Liu, Y., and Favier, D. (2011). Assessment of tension-compression asymmetry of NiTi using circular bulge testing of thin plates. Scripta Materialia, 65(4), 347-350.

17. Dudderar, T., Koch, F., and Doerries, E. (1977). Measurement of the shapes of foil bulge-test samples. Exp. Mech., 17, 133-140. 
18. Wineman, A. S. (1976). Large axisymmetric inflation of a nonlinear viscoelastic membrane by lateral pressure. Trans. Soc. Rheo., 20(23), 2031976.

19. Yang, W. and Feng, W. (1970). On axisymmetrical deformations of nonlinear membranes. $J$. Appl. Mech., 37, 1002-1011.

20. Klingbeil, W. and Shield, R. (1964). Some numerical investigations on empirical strain energy functions in the large axisymmetric extensions of rubber membranes. Z. Angew. Math. Phys., 15, 608-629.

21. Wineman, A. S. (1978). On axisymmetric deformations of nonlinear viscoelastic membranes. J. Non-Newtonian Fluid Mech., 4(23), 249-260.

22. Feng, W. (1992). Viscoelastic behavior of elastomeric membranes. J. Appl. Mech., 59, 29-34.

23. Hsu, F., Liu, A., Downs, J., Rigamonti, D., and Humphrey, J. (1995). A triplane video-based experimental system for studying axisymmetrically inflated biomembranes. IEEE T. Bio-Med. Eng., 42, 442-450.

24. Luo, P., Chao, Y., Sutton, M., and Peters, W. (1993). Accurate measurement of threedimensional deformations in deformable and rigid bodies using computer vision. Exp. Mech., 33, 123-132.

25. Becker, T., Splitthof, K., Siebert, T., and Kletting, P. (2006). Error estimations of 3D digital image correlation measurements. Proc. of SPIE, 6341, 63410F. doi: 10.1117/12.695277

26. Sutton, M. A. (2008). Digital image correlation for shape and deformation measurements. Springer Handbook of Experimental Solid Mechanics - PartC, pages 565-600.

27. Sutton, M. A., Orteu, J.-J., and Schreier, H. W. (2009). Image Correlation for Shape, Motion and Deformation Measurements: Basic Concepts, Theory and Applications. Springer.

28. Orteu, J. J. (2009). 3-D computer vision in experimental mechanics. Opt. Laser Eng., 47(3-4), 282-291.

29. Schreier, H. and Sutton, M. (2002). Systematic errors in digital image correlation due to undermatched subset shape functions. Exp. Mech., 42, 303-310.

30. Carmo, M. P. (1976). Differential geometry of curves and surfaces. Prentice-Hall lnc., Englewood Cliffs, New Jersey.

31. Ciarlet, P. (2005). An Introduction to Differential Geometry with Applications to Elasticity. Springer. 
32. Toponogov, V. A. (2006). Differential Geometry of Curves and Surfaces: A Concise Guide. Boston, MA.

33. Green, A. and Adkins, J. (1970). Large Elastic Deformation. Clarendon Press - Oxford, 2nd edition.

34. Humphrey, J. D. (1998). Computer methods in membrane biomechanics. Comput. Methods Biomech. Biomed. Eng., 1, 171- 210.

35. Mooney, M. (1940). A theory of large elastic deformation. J. Appl. Phys., 11, 582-592.

36. Kyriacou, S. K., Shah, A. D., and Humphrey, J. D. (1997). Inverse finite element characterization of nonlinear hyperelastic membranes. J. Appl. Mech., 65, 257-262.

37. Garcia, D. (2010). Robust smoothing of gridded data in one and higher dimensions with missing values. Comput. Statist. Data Anal., 54(4), 1167 - 1178.

38. Orteu, J.-J., Bugarin, F., Harvent, J., Robert, L., and Velay, V. (2010). Multiple-camera instrumentation of a single point incremental forming process pilot for shape and 3D displacement measurements: Methodology and results. Exp. Mech., 51, 1-15.

39. Machado, G., Chagnon, G., and Favier, D. (2010). Analysis of the isotropic models of the mullins effect based on filled silicone rubber experimental results. Mech. Mater., 42(9), 841 - 851.

40. Mullins, L. (1969). Softening of rubber by deformation. Rubber Chem. Technol., 42, 339-362.

41. Treloar, L. R. G. (1943). The elasticity of a network of long chain molecules (I and II). Trans. Faraday Soc., 39, 36-64; 241-246.

42. Guélon, T., Toussaint, E., Le Cam, J.-B., Promma, N., and Grédiac, M. (2009). A new characterisation method for rubber. Polym. Test., 28(7), $715-723$. 